Ann. Génét. Sél. anim., I978, 10 (3), 443-474.

\title{
Analyse des liaisons entre la morphologie et l'aptitude au galop au trot et au saut d'obstacles chez le Cheval
}

\author{
B. LANGLOIS, J. FROIDEVAUX, L. LAMARCHE, C. LEGAULT \\ P. LEGAULT $\left({ }^{*}\right)$, Luce TASSENCOURT $\left({ }^{* *}\right)$ et M. THÉRET $\left(^{*}\right)$ \\ Station de Génétique Quantitative et Appliquée \\ Centre national de Recherches zootechniques, I.N.R.A. \\ 78350 Jouy-en-Josas \\ (*) École Nationale Vétérinaive d'Alfort, 947 ro Maisons-Alfort \\ (**) Département de Biométrie du C.N.R.Z., 78350 Jouy-en-Josas
}

\begin{abstract}
Résumé
Pour obtenir rapidement un nombre élevé de mensurations par cheval nous avons été amenés à perfectionner et tester une méthode photométrique. L'étude de la répétabilité d'une trentaine de mesures effectuées sur 6o chevaux a montré que selon les cas 2 ou 3 photographies permettaient d'atteindre une précision satisfaisante. Par ailleurs des analyses en composantes principales ont permis de réduire les nombreuses mensurations relevées à 4 facteurs essentiels. Ce sont la taille (40 p. roo de la variance totale), la compacité ( 12 p. roo), l'orientation des rayons postérieurs ( 7 p. I0o) et l'orientation des rayons antérieurs $(6 \mathrm{p}$. 100).

L'étude des différences entre Trotteur Français et Pur Sang Anglais a été co:Iduite par cette méthode sur respectivement 40 et 42 animaux à l'aide de $2 \mathrm{I}$ variables. L'analyse de la variance et la recherche d'une fonction discriminante entre ces deux populations a permis de préciser l'étendue des divergences morphologiques induites dans ces deux races par la sélection sur les performances. Les Trotteurs se distinguent par des angles de l'ilium, du fémur et du scapulum avec l'horizontale plus élevée. Le radius est plus court et le canon très notablement plus épais. En outre, de taille comparable aux Pur Sang Anglais, ils sont plus larges et par conséquent plus compacts.

La liaison entre la morphologie et l'aptitude aux sauts d'obstacles chez le cheval de sport a été étudiée sur deux échantillons indépendants :

- d'une part 94 étalons des haras nationaux ayant un indice génétique sur descendance en concours de sauts d'obstacles dont le coefficient de détermination est supérieur ou égal à 0,40 ;

- d'autre part 103 chevaux répartis en "Bons" et "Mauvais sauteurs " choisis dans 26 centres équestres.

Sur le premier échantillon l'explication de l'indice génétique par régression multiple progressive est assez décevante. Au seuil de 5 p. roo interviennent seulement les fortes valeurs de la hauteur au garrot pour l'ensemble des races (94 étalons) et pour les Selle Français (45 étalons), la fermeture de l'angle de l'humérus avec l'horizontale pour les $A$ nglo-Arabes (28 étalons), l'ouverture de l'angle de l'ilium avec l'horizontale pour les Pur Sang Anglais (2x étalons).

Sur le second échantillon l'effet de l'aptitude a été mis en évidence par une analyse de la variance à deux voies (sexe-aptitude). Neuf variables diffèrent significativement. Cela se traduit pour les "Bons sauteurs" par rapport aux "Mauvais " par les largeurs aux épaules, aux trochanters et aux hanches plus grandes, un périmètre thoracique plus long, un ilium plus grand et un fémur plus incliné sur l'horizontale, un scapulum et un humérus plus allongés, leurs angles avec l'horizontale étant plus fermés.
\end{abstract}


Alors que 1'analyse discriminante permettait de distinguer facilement un trotteur d'un pur sang (5 p. Ioo de déclassés) elle souligne la difficulté qu'il y a à distinguer un "Bon sauteur " d'un "Mauvais" (27 p. roo de déclassés). Des tendances peuvent toutefois être dégagées de l'ensemble des calculs. Le sauteur est un cheval plutôt grand, très compact (large et près de terre), avec une croupe longue, large et fortement inclinée dont. les angles des autres rayons moteurs (scapulum, humérus et fémur) sont fermés sur l'horizontale.

Pour vérifier l'efficacité des concours "modèle et allures " pour la sélection d'un cheval de sport les performances en épreuves de saut d’obstacles de 645 chevaux présentés au concours régional de Saint-Lo de I97I à I975 ont ensuite été comparées à leur classement " au modèle ". Il ressort de cette étude que ces concours ne permettent de déterminer que de l'ordre de $4 \mathrm{p}$. Ioo de la valeur génétique en saut d'obstacles alors que les propres performances estimées par les gains en expliquent de l'ordre de $20 \mathrm{p}$. Ioo et le contrôle de la descendance des étalons $60 \mathrm{p}$. I00 en moyenne.

On conclut pour améliorer l'aptitude au saut d'obstacles à la supériorité du contrôle de performance sur une sélection indirecte sur la conformation.

\section{Introduction}

Apprécier à sa juste valeur le modèle d'un cheval suppose une éducation longue et approfondie qui comporte souvent un facteur personnel important. C'est un art accessible à de rares privilégiés. L'idéal est en effet d'obtenir des observations objectives résultant de constatations indépendantes de l'observateur. C'est pour cela que progressivement est née la notion "d'hippométrie ". Elle permet d'exprimer par des chiffres et des rapports les jugements du " connaisseur ", de confirmer ou rectifier des impressions visuelles, de fixer des normes pour la production d'un type bien défini, de réaliser enfin une unité de vue dans le temps entre les jurys chargés du choix des reproducteurs.

Depuis l'existence de l'hippométrie, les résultats escomptés n'ont pas été entièrement obtenus. Des calculs de proportion, d'indices corporels ont été entrepris mais aucun n'a eu d'application pleinement satisfaisante. Il semble en effet que le manque de moyens de calcul puissant ait été le principal obstacle au développement et à la généralisation de cette technique. Depuis, les conditions ont bien changé. L'utilisation des méthodes statistiques multivariates est rendue possible grâce à l'informatique. Les problèmes liés à l'hippométrie se présentent donc sous un jour différent. Il nous a alors paru intéressant de reprendre ces travaux afin de préciser de façon objective les liaisons entre la morphologie et différentes aptitudes recherchées chez le cheval. C'est ainsi que nous avons été amenés à perfectionner une méthode de mensuration pour l'appliquer ensuite d'une part à l'étude des différences entre Trotteur Français et Pur Sang Anglais, races sélectionnées de longue date sur des performances divergentes et d'autre part pour préciser les composantes du modèle du "sauteur ", connaissance de première importance pour la sélection du cheval de sport. Parallèlement, l'estimation de l'efficacité du " concours modèle et allures " pour la sélection du cheval d'obstacle a été réalisée. Nous exposerons donc ici la synthèse de différents travaux, les mémoires de fin d'étude de FroIDEVAux (I975) et de LaMARChe (1977) et la thèse vétérinaire de $L_{E G A U L T}$ (I977) qui ont été réalisés dans le cadre de la convention Haras-I.N.R.A. Des informations plus détaillées sont fournies dans les textes originaux dont les références sont en annexe. 


\section{I. - Matériel et méthodes}

\section{A. - Méthode de mensuration}

Les techniques classiques utilisent des instruments tels que la toise pour les hauteurs, la canne de LYNDTIN (toise à deux potences) pour les longueurs, le compas d'épaisseur (sorte de pied à coulisse géant) pour les largeurs, le ruban métrique pour les circonférences, le pied à coulisse pour des mesures plus fines. Par ailleurs 1'hippométrie a développé ses propres instruments : 1e "saumurien " sorte de mètre ruban plombé coulissant dans une plaque pour la mesure du vide sous-sternal ou " l'arthrogoniomètre " (LEMOIGNE, I877) pour la mesure des angles articulaires en sont des exemples.

La manipulation rapide de l'ensemble de ces instruments afin de relever sur un cheval dans un temps minimum un maximum de mensurations fiables, ne nous a pas paru aisée. Aussi nous sommes nous penchés sur une méthode photographique proposée pour évaluer les angles articulaires par GoUBAUX et BARRIER (I884). Les progrès des techniques aidant (diapositives, possibilité importante d'agrandissement...) nous avons pu l'étendre à l'estimation des mensurations latérales de l'animal, les mesures de largeur ou d'épaisseur étant prises de façon classique (compas d'épaisseur, ruban métrique, pied à coulisse). En effet en matérialisant des points de repère du squelette à l'aide de tissu adhésif (carré de sparadrap blanc ou rose suivant la robe) le cheval est photographié au télé objectif. Un aide tient un " mètre étalon " dans le plan de l'animal, qui fournira l'échelle de référence pour les calculs ultérieurs. La dispositive obtenue est ensuite projetée sur un écran où les pastilles sont pointées. Ces points reliés les uns aux autres par des droites donnent une épure du cheval. Les mesures d'angle sont alors faites au rapporteur et par l'intermédiaire de la toise les distances mesurées à la règle sont converties en vraie grandeur (fig. I et 2 ).

Le choix des points de repère détermine la nature des mensurations relevées, la méthode offrant à l'opérateur une large gamme de mesures simultanées. D'un opérateur à l'autre des variations pourront intervenir mais l'essentiel est l'obtention d'une bonne régularité au sein d'une même étude.

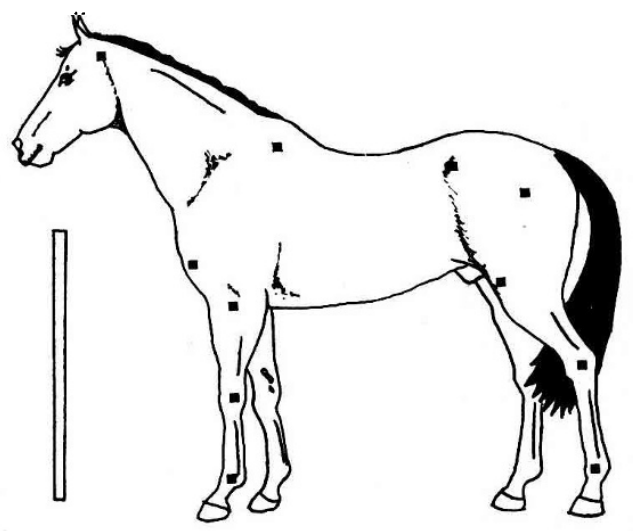

Frg. I. - Cheval prêt à être photographié. Horse ready for being photographed. 


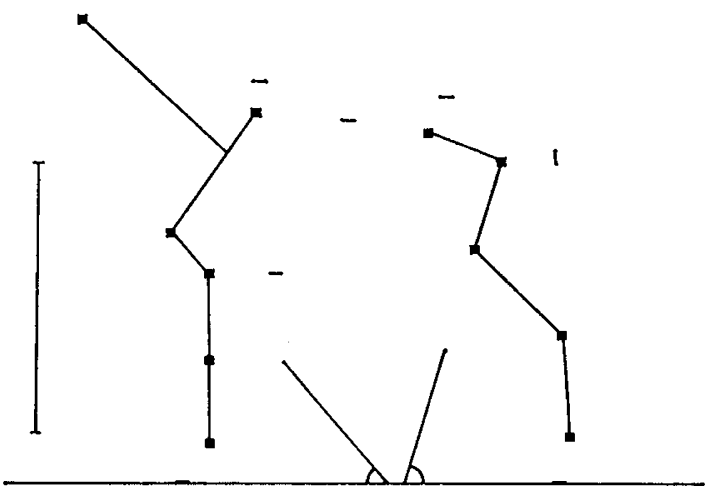

FIG. 2. - Épure du cheval après projection.

Diagram of the horse after projection.

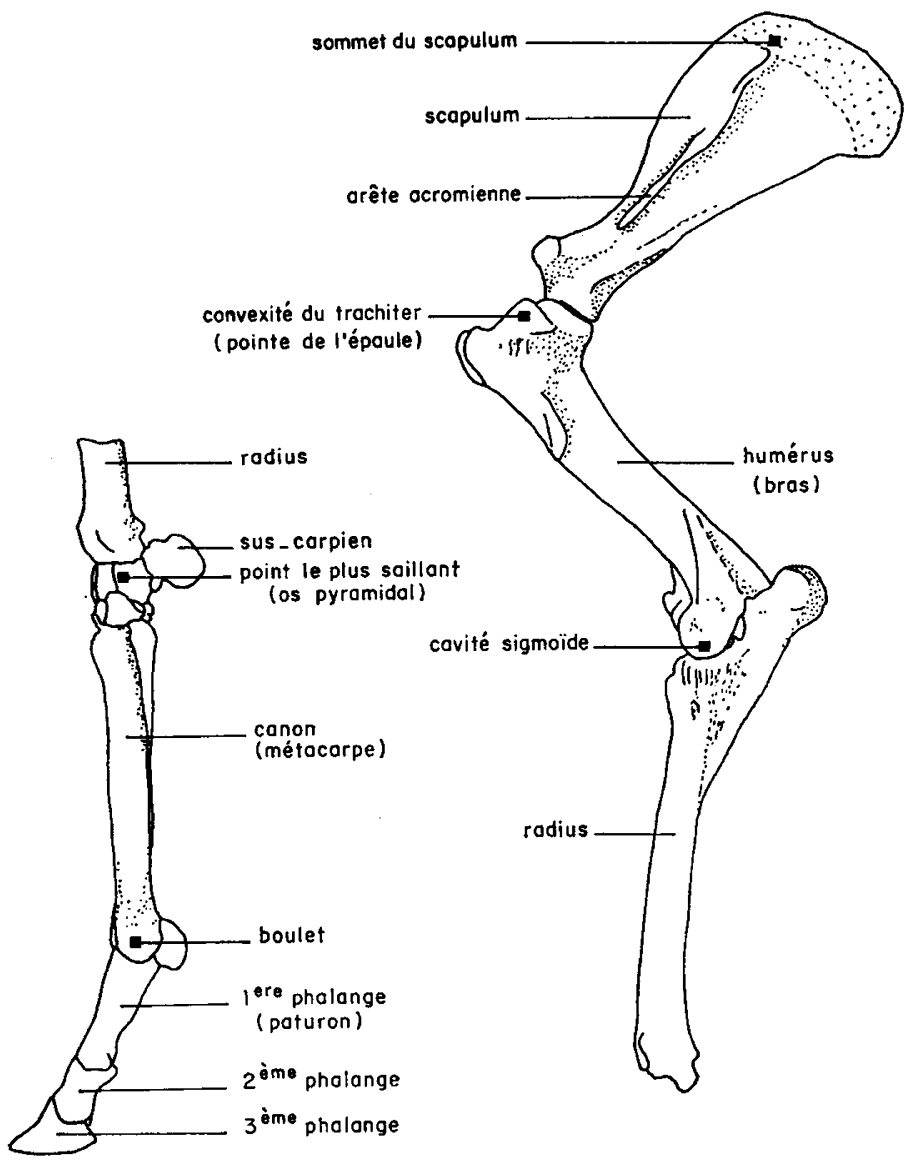

FIG. 3. - Position des repères sur le membre antérieur.

Positions of the marks on the foreleg. 
D'une façon générale nous recommandons d'utiliser les points de repère définis par LEMOIGNE (I877) comme représentatifs des axes de rotation des différents rayons des membres:

\section{- membre antérieur (fig. 3)}

" sommet $d u$ scapulum "

A l'extrémité du scapulum dans le prolongement de l'épine acromienne. On suivra l'arête osseuse avec le doigt et on placera la pastille adhésive à l'endroit où le doigt plonge brusquement.

"axe scapulo-huméral"

Son point de repère externe est situé à peu près au niveau de la convexité du trochiter. Sur l'humérus on ressent au palper trois sommets deux sont en avant et forment la pointe de l'épaule, le troisième qui sert de référence se situe en arrière dans le prolongement vers le bas de l'épine acromienne.

\section{"axe huméro-radial "}

Son point de repère externe se situe à l'insertion humérale du ligament latéral externe de cette jointure. A partir de la coulisse tendineuse on remonte verticalement entre le radius et l'olécrâne jusqu'à la cavité sigmoïde.

"axe radio-carpien "

A un centimètre au-dessous de la tubérosité externe et inférieure du radius. Pratiquement ce point se situe à l'endroit le plus saillant vers l'extérieur du genou.

"axe métacarpo-phalangien"

A l'insertion supérieure du ligament latéral correspondant de l'articulation. Le point de repère se trouve également à l'endroit le plus saillant du boulet mais situé dans une petite fossette.

- membre postérieur (fig. 4)

"pointe de la hanche"

Sur la tubérosité inférieure et postérieure de l'épine iliaque antérieure.

"axe coxo-fémoral "

Point de repère externe un peu au-dessous et en arrière de la convexité du trochanter. Lorsque aux $2 / 3$ environ de l'axe pointe de la hanche - pointe de la fesse, on explore les muscles de la croupe on ne tarde pas à sentir en dessous une aire de quelques centimètres carrés correspondant à la convexité du trochanter.

" axe fémoro-tibial"

Un peu au-dessous de l'insertion supérieure du ligament latéral correspondant de cette jointure. Pour être visible le point de repère doit être posé plus en arrière, le pli du grasset pouvant servir de guide on le pose alors au sommet du tibia.

"axe tibio-tarsien"

$\mathrm{Au}$ centre de 1'astragale. Le point se pose donc au centre du jarret.

"axe métatarso-phalangien"

A l'insertion du ligament latéral correspondant. Le point de repère est identique à celui du membre antérieur. 

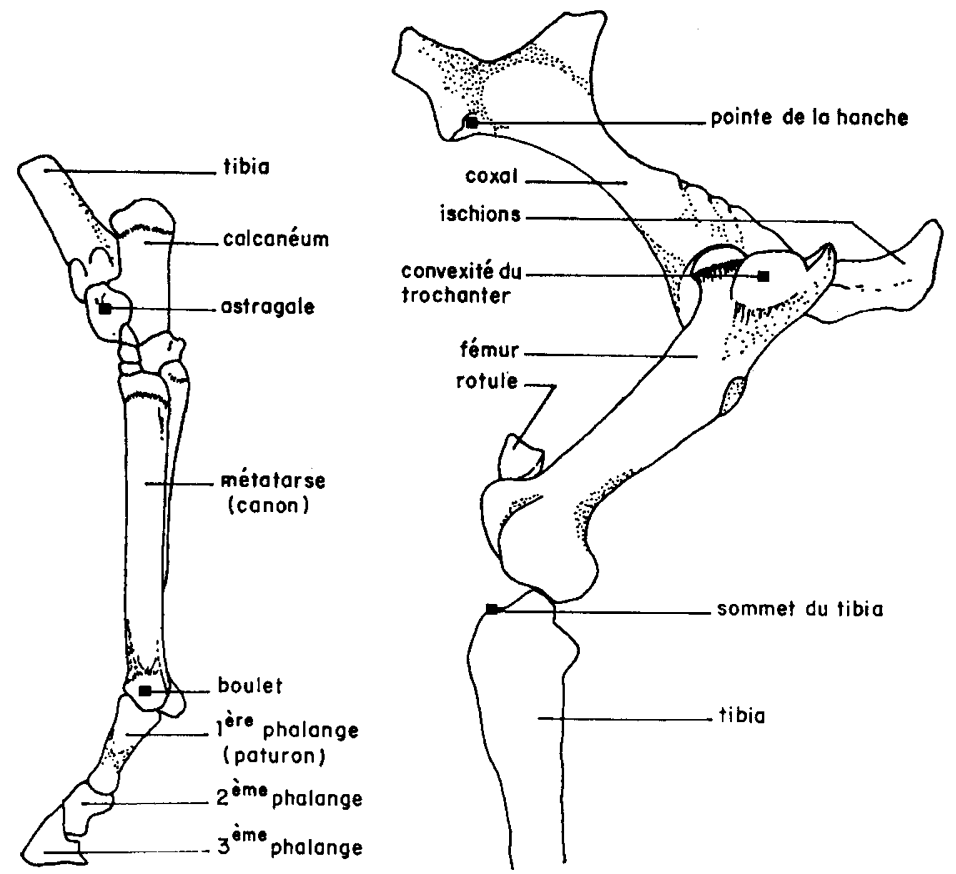

FIG. 4. - Position des repères sur le membre postérieur.

Positions of the marks on the hindleg.

Cette liste n'est pas limitative d'autres repères peuvent être envisagés, l'apophyse zygomatique (tête), le sommet de l'oléocrane (coude), la dernière côte... D'autres encore sont visibles immédiatement sur la photographie. Il s'agit tout d'abord des points de contact des sabots avec le sol qui fournissent l'horizontale de référence pour la mesure des angles et des hauteurs. De même, en pointant le profil du cheval la hauteur au garrot, la hauteur au dos (au point le plus bas), la hauteur à la croupe (au point le plus haut), le vide sous-sternal (au niveau du passage de sangle) et la longueur de la pointe de l'épaule à la pointe de la fesse peuvent être estimés. L'évaluation de la longueur de l'encolure a été obtenue entre un point fixé arbitrairement au I/3 supérieur du scapulum et 1'apophyse zygomatique.

Ces différents repères fixés donnent naissance à une multitude de mesures. Nous ne décrirons pas ici les variables retenues car elles apparaissent clairement dans les résultats.

\section{B. - Matériel animal}

Afin de tester notre méthode de mensuration, 60 chevaux de taille, de modèle et d'origine variés, répartis dans trois centres équestres de la région parisienne, ont été soumis deux fois à notre protocole à plusieurs jours d'intervalle. L'étude de cet échantillon a permis d'estimer la répétabilité de 37 mesures différentes et par l'examen de leurs corrélations de faciliter pour la suite le choix d'un certain nombre d'entre elles. 
L'étude des différences entre Trotteur Français (TF) et Pur Sang Anglais (PSA) a été conduite sur respectivement 40 et 42 animaux à 1'aide de $2 \mathrm{I}$ variables. Des chevaux de réforme, des étalons, quelques poulinières et une majorité de chevaux à l'entraînement ont constitué cet échantillon. Il en est résulté une disproportion en faveur des mâles (33 pour les PSA et 25 pour les TF). Par ailleurs les femelles de Pur Sang Anglais ont paru un peu hypermétriques.

Pour préciser les relations entre la conformation et l'aptitude au saut, deux échantillons ont été étudiés à l'aide de 30 variables.

- D'une part 94 étalons nationaux (42 Selle Français, 28 Anglo-Arabes, 2I Pur Sang Anglais et 3 Trotteurs Français) ont été mesurés et photographiés dans I4 haras. Ils avaient tous un indice de sélection sur descendance en Concours de Sauts d'Obstacles (LANGLOIS, I977) de coefficient de détermination supérieur à 0,40 (au moins I2 descendants gagnants). Les relations entre conformation et aptitude à produire des gagnants pourront ainsi être mises en évidence.

- D'autre part 76 mâles et 27 femelles répartis par moitié entre " bons sauteurs " et " mauvais sauteurs " (respectivement 52 " $B$ ) et $5 \mathrm{I}$ " $\mathrm{M}$ )) ont été mesurés dans 26 établissements. Le choix était fait dans les clubs hippiques et chez les cavaliers professionnels avec 1'aide du responsable. Les individus qualifiés " $B$ ") sont ceux qui paraissent les plus doués non seulement par leur puissance mais aussi par leur style. La plupart de ces sujets ont fait leurs preuves dans les compétitions officielles. Une quinzaine d'entre eux se sont classés en épreuves internationales. Les individus qualifiés " $M$ » sont les chevaux de l'établissement les plus naturellement inaptes. Ils sont toutefois en bonne santé et en bon état " psychique ". Les différences morphologiques entre ces deux groupes pourront être appréhendées et des recoupements réalisés avec les résultats obtenus sur les étalons.

Enfin, il nous fallait vérifier si les concours de " modèle et allures " avaient une efficacité satisfaisante pour la sélection d'un cheval de sport pour le Concours de Sauts d'Obstacles. Nous nous sommes donc attachés à suivre le devenir en compétition des chevaux de Selle Français présentés à trois ans au concours régional de Saint-Lô de I97I à I975. Ces listes étaient publiées chaque année dans le Bulletin de la Société Hippique Française. Disposant parallèlement de 1'indice génétique sur performance individuelle de tous les chevaux ayant gagné de l'argent en Concours de Sauts d'Obstacles de I972 à I976 (LANGLOIS, I977), il nous a été possible de faire les rapprochements nécessaires. 645 chevaux au total ont permis la comparaison. La moyenne du coefficient de détermination de leurs indices était de 0,22 et ils représentaient 39 p. Ioo des individus classés à Saint-Lô.

\section{C. - Méthodes statistiques}

\section{Analyse de la variance}

Cette méthode a été utilisée pour l'estimation de la répétabilité des mesures et pour l'étude des différences entre groupes.

Dans le premier cas le modèle aléatoire suivant a été utilisé :

$\mathrm{X}_{i j}=\mu+c_{i}+r_{i j}$

$\mathrm{X}_{i j} \quad$ est la mesure $j$ du cheval $i$

$\mu \quad$ est la moyenne générale

$c_{i} \quad$ est 1'effet du cheval $i$

$r_{i j} \quad$ est la résiduelle.

La fraction de variance due au cheval estime la répétabilité de la mesure. 
Dans le second cas, comparaison du Trotteur Français au Pur Sang Anglais ou des "Bons sauteurs" aux "Mauvais " en tenant compte de l'effet du sexe, un modèle à effets fixés exprimant une éventuelle interaction a été utilisé :

$\mathrm{X}_{i j k}=\mu+\mathrm{S}_{i}+\mathrm{A}_{j}+\mathrm{I}_{i j}+r_{i j k}$.

$\mathrm{X}_{i j k}$ est la valeur du cheval $k$ de sexe $i$ et d'aptitude $j$ (ou race $j$ ).

$\mu \quad$ est la moyenne générale.

$\mathrm{S}_{i} \quad$ est l'effet du sexe $i$ ( 2 niveaux).

$A_{j}$ est l'effet de l'aptitude $j$ (ou race $j$ ) ( 2 niveaux).

$\mathrm{I}_{i j} \quad$ est 1'effet d'interaction entre le sexe $i$ et 1'aptitude $j$ (ou race $i$ ) (4 niveaux).

$r_{i j k}$ est la résiduelle.

Analyse en composantes principales

C'est une analyse des $n \frac{n-\mathrm{I}}{2}$ coefficients de corrélations de $n$ variables entre elles (435 pour 30 critères). Elle vise à condenser l'information de départ en de nouvelles variables combinaisons linéaires des précédentes, les composantes principales ou facteurs. Elle a le grand avantage de simplifier l'examen de nombreuses corrélations en ne considérant qu'un effectif limité de facteurs indépendants représentant chacun une association de critères étroitement liés entre eux. L'examen des corrélations des anciennes variables avec les nouvelles permet de les interpréter. Par ailleurs en ne considérant que le nombre limité des composantes principales on peut plus facilement réaliser les groupements d'observations et des représentations graphiques sont envisageables, le nombre d'axes à prendre en considération étant réduit.

\section{Analyse discriminante à deux populations}

Lorsque l'on dispose de $n$ variables sur deux populations cette analyse établit la combinaison linéaire qui tend à maximiser les différences entre ces deux ensembles. On obtient ainsi la fonction discriminante de Fisher $(z)$. On en déduit une mesure de ressemblance entre les deux populations en calculant la distance de Mahalanobis $\left(\mathrm{D}^{2}\right)$ qui les sépare. Il s'agit de la généralisation à une combinaison linéaire de $n$ variables d'un test " $t$ " pour un seul critère. L'étude de la fonction discriminante $(z)$ permet de situer a posteriori un cheval $(x)$ dans l'une ou l'autre des populations de départ.

$z>$ o $x$ appartient à la première population.

$z<0 x$ appartient à la seconde population.

Cela permet d'apprécier l'efficacité de la discrimination par le pourcentage d'animaux déclassés (animaux de la première population classés dans la seconde et vice versa). On peut aussi calculer a posteriori les probabilités pour chaque individu d'appartenir à l'une ou l'autre des deux populations et en tracer les histogrammes. L'allure bimodale résultant d'une zone de chevauchement plus ou moins forte fournira la même indication.

On peut affiner l'analyse en introduisant les variables pas à pas. La première variable introduite est celle qui permet la plus grande discrimination. Elle a le $\mathrm{D}^{2}\left(t^{2}\right)$ le plus élevé. Puis on introduit la seconde, celle qui associée à la première permet la meilleure discrimination à deux variables. On poursuit ainsi l'analyse jusqu'à introduction de toutes les variables. On peut également, c'est ce que nous avons fait, fixer un seuil a priori pour l'introduction ou l'exclusion des variables; on ne conserve ainsi que celles qui apportent une contribution significative à la discrimination. 


\section{Régression multiple progressive}

Pour les étalons, les relations entre les 30 mesures prises et l'indice génétique sur descendance ont été étudiées par la technique de la régression multiple progressive. On cherche donc la combinaison linéaire des écarts de chacune des variables à sa moyenne qui a la corrélation maximum avec l'indice génétique. Comme précédemment les variables peuvent être introduites pas à pas. I a première est celle qui au départ prédit le mieux l'indice. La suivante est celle qui, compte tenu de la (des) précédente(s) apporte le plus d'explication de la variable prédite. Lorsqu'une variable est introduite, elle peut remettre en cause la signification de celles figurant déjà dans la régression. Nous avons choisi arbitrairement d'étudier les variables au moment où le plus grand nombre d'entre elles apportent une contribution significative à l'explication de l'indice. Ce moment se situe le plus souvent au début du plateau de détermination lorsque l'introduction de nouvelles variables sans augmenter véritablement la détermination globale semble nuire à la signification des précédentes. En effet au palier de détermination, 1'introduction de nouveaux critères entraîne un " phénomène de brouillage " qui masque les variables significatives, d'une part du fait de la prise en compte de déviations aléatoires non significatives des corrélations et d'autre part d'une certaine redondance dans l'information.

L'introduction des variables avec un seuil fixé a priori ne permet d'introduire que des critères dont la contribution à la détermination est significative. C'est la seule démarche permettant de déboucher sur des équations de prédiction correctes. Mais étant beaucoup plus exigeante au niveau de chaque variable, elle n'en introduit qu'un nombre limité et ne permet pas une analyse aussi fine que la précédente.

\section{Étude des rangs}

La confrontation des résultats de " modèle et allures " à ceux des compétitions posait un certain nombre de problèmes. Deux méthodes ont été utilisées pour les résoudre :

\section{- la corrélation de rang:}

pour chaque classement sur le modèle, les chevaux communs à nos deux fichiers (modèle et indices) ont été ordonnés respectivement selon ces deux critères. Les corrélations de rang $\left(r_{s}\right)$ ont ainsi été réalisées intra concours selon SpEARMAN (I904);

\section{- la corrélation simple:}

à chaque rang de modèle il était également possible d'affecter en fonction de l'effectif d'animaux classés une déviation moyenne d'une variable normale centrée réduite. Ces valeurs tabulées pour des effectifs allant jusqu'à Ioo (PEARSON et HARTLEY, I972) ont donc permis de calculer une corrélation classique $(r)$ avec l'indice génétique.

Une estimation de la corrélation moyenne pour les 26 classements considérés, a été conduite simultanément à un test d’homogénéité selon LUSH (I93I).

\section{- Méthodes statistiques et génétiques}

L'étude des corrélations de variables morphologiques avec un indice génétique appelle les remarques suivantes : 
Si l'on considère que l'indice génétique $X_{1}$ et l'appréciation du modèle $X_{2}$ constituent deux estimateurs indépendants de la même valeur génétique en Concours de Sauts d'Obstacles Y, on peut écrire la relation suivante entre les corrélations :

$r\left(\mathrm{X}_{1}, \mathrm{X}_{2}\right)=r\left(\mathrm{X}_{1}, \mathrm{Y}\right) \times r\left(\mathrm{X}_{2}, \mathrm{Y}\right)$

$r\left(\mathrm{X}_{1}, \mathrm{X}_{2}\right)$ peut être mesuré expérimentalement

$r\left(\mathrm{X}_{1}, \mathrm{Y}\right)$ corrélation entre l'indice génétique et la valeur génétique est égale à la racine carrée du coefficient moyen de détermination

$r\left(\mathrm{X}_{2}, \mathrm{Y}\right)$ généralement inconnu peut ainsi être estimé.

La comparaison de $r\left(\mathrm{X}_{1}, \mathrm{X}\right)$ à $r\left(\mathrm{X}_{2}, \mathrm{Y}\right)$ permet de conclure, sous réserve que les effectifs soient satisfaisants, à la plus ou moins grande efficacité de la sélection sur $X_{1}$ ou sur $X_{2}$ pour l'amélioration génétique de l'aptitude au Concours de Sauts d'Obstacles $(Y)$. Soulignons toutefois que si $X_{1}$ et $X_{2}$ ne sont pas conditionnellement indépendants, c'est-à-dire que seule leur liaison à $\mathrm{Y}$ est supposée contribuer à leur corrélation, l'estimation de $r\left(\mathrm{X}_{2}, \mathrm{Y}\right)$ se trouve sur évaluée. Cette estimation a donc tendance à fournir des valeurs maximales.

\section{II. - Résultats}

\section{A. - Estimation de la répétabilité des mesures}

Le tableau I donne les estimations de la répétabilité et les caractéristiques moyennes des 60 chevaux de 1'échantillon pour 37 variables. Les valeurs trouvées supérieures à 0,70 dans l'ensemble, sont cependant plus faibles pour les mesures qui font intervenir la cavité sigmoïde et surtout la rotule. L'évaluation des angles quant à elle paraît moins répétable que celle des distances.

En veillant tout particulièrement au positionnement précis du point de repère au niveau de la rotule et à une présentation du cheval bien d'aplomb (ni campé, ni assis) ces valeurs nous montrent que selon les mensurations désirées deux ou trois photographies de l'animal sont suffisantes pour atteindre une précision satisfaisante.

\section{B. - Analyses de la variance portant sur les effets sexe et aptitude (ou race)}

Les résultats concernant les différences entre Pur Sang Anglais et Trotteur Français sont reportés tableaux 2, 3 et 4 . Remarquons simplement pour l'instant que la hauteur entre croupe et trochanter, la longueur de la pointe de l'épaule à la hanche, les largeurs aux épaules et aux hanches, l'épaisseur du canon, les angles du scapulum, de l'ilium et du fémur avec l'horizontale sont significativement plus faibles chez les Pur Sang Anglais. Pour ces galopeurs seule la longueur du radius est significativement plus importante. Par ailleurs une interaction entre race et sexe est apparue pour la longueur de l'humérus et l'épaisseur du canon, les femelles Pur Sang Anglais de l'échantillon paraissant particulièrement développées pour ces caractères. 


\section{TABLEAU I}

Estimation de la répétabilité des mesures faites sur 60 chevaux de service Estimation of the repeatability of measurements made on 60 stock horses

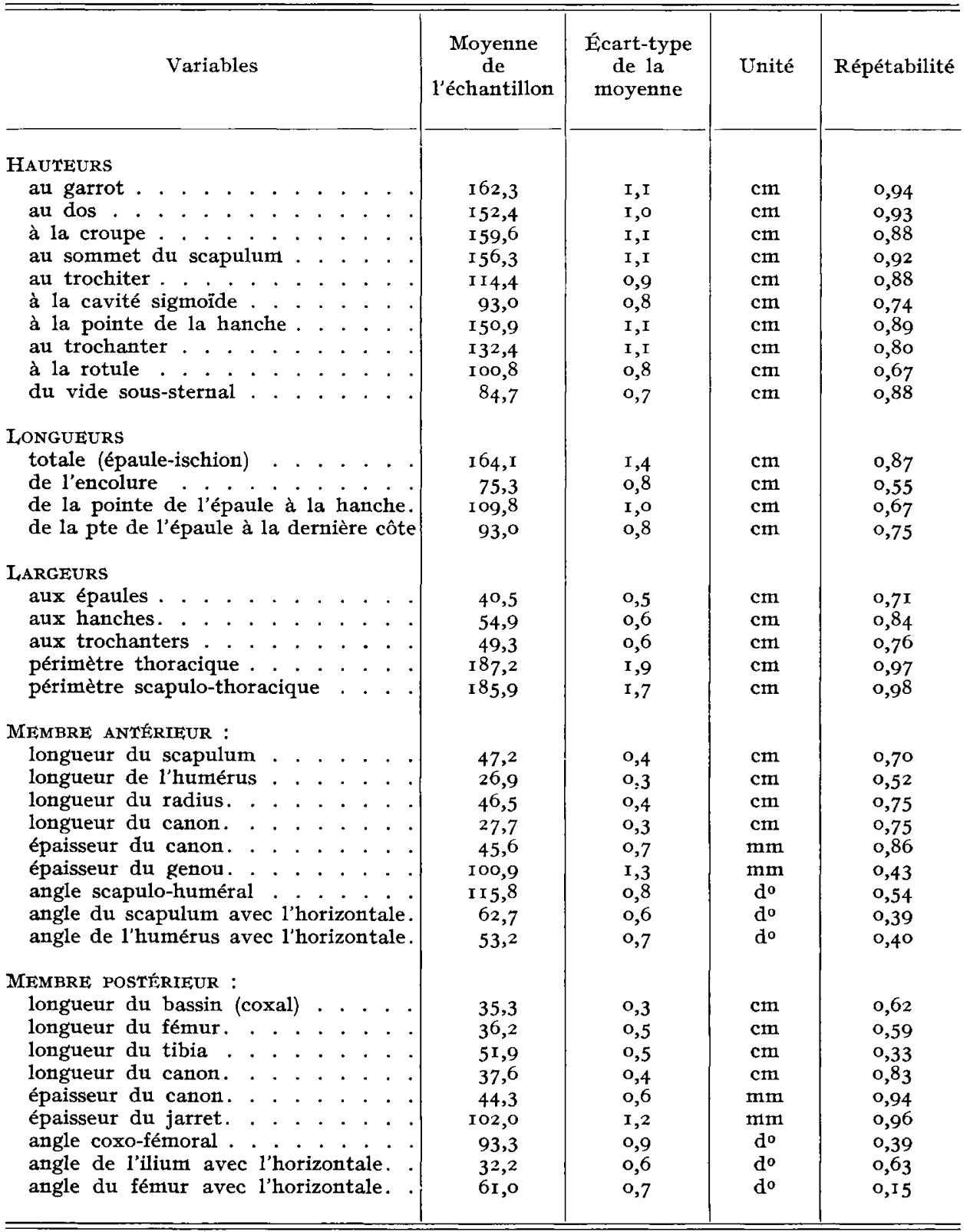


TABLEAU 2

Résultats de l'analyse de la variance : signification des effets sur les 40 trotteurs et 42 Pur Sang

Analysis of variance: significance of sex and breed effects on 40 Trotters and 42 Thoroughbreds

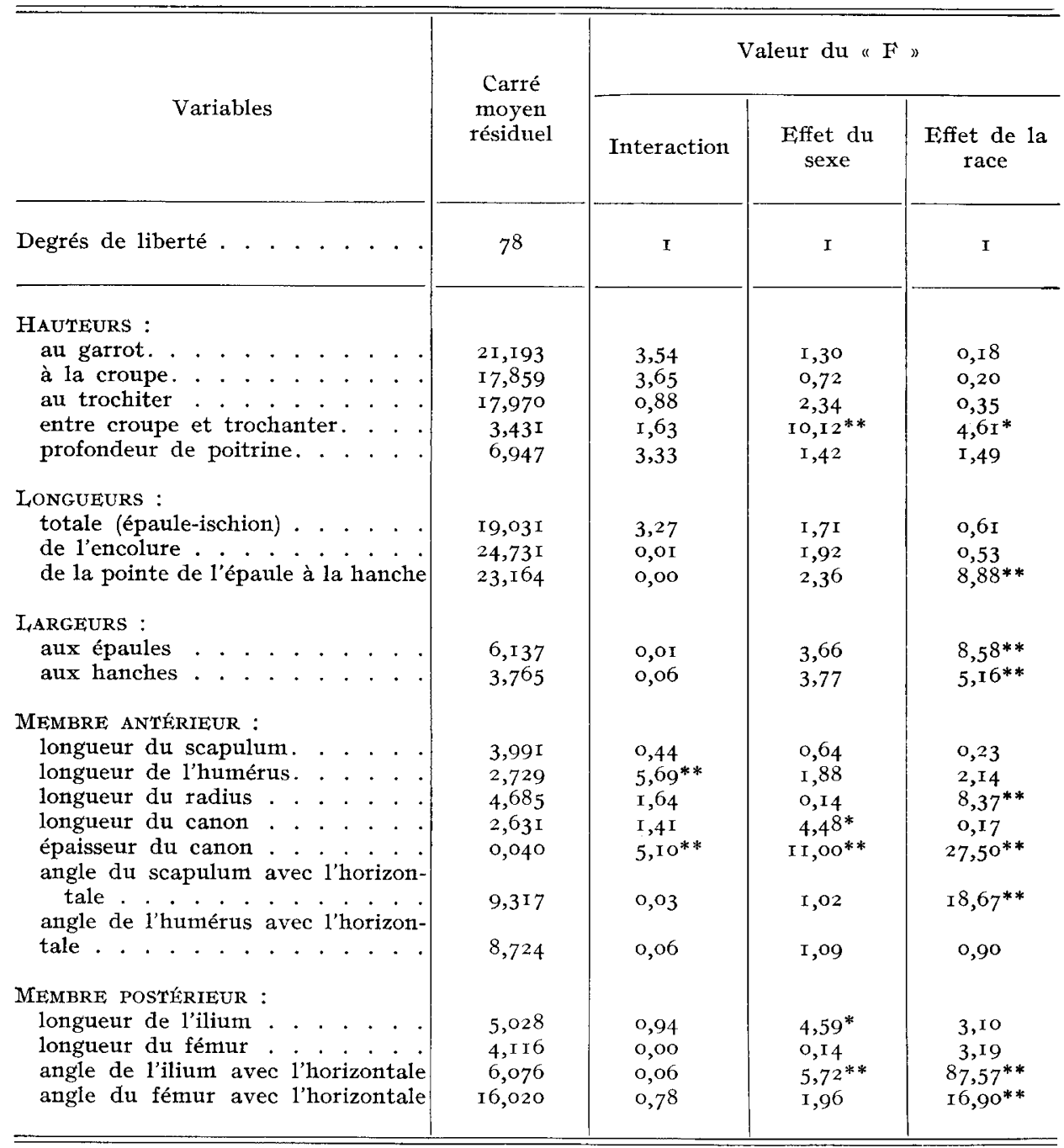

Les résultats concernant 1'analyse des différences entre "Bons " et " Mauvais sauteurs " sont reportés tableaux 5 et 6 . Les "Bons sauteurs " sont significativement plus larges (largeurs aux épaules, aux hanches, aux trochanters et périmètre thoracique), ont le scapulum, l'humérus et l'ilium plus longs, l'angle du scapulum et du fémur avec l'horizontale étant plus fermé. 
TABLEAU 3

Résultats de l'analyse de la variance

estimées des effets " sexe "et a race " sur les 40 trotteurs et les 42 Pur Sang

Analysis of variance

estimates of sex and breed effects on 40 trotters and 42 thoroughbreds

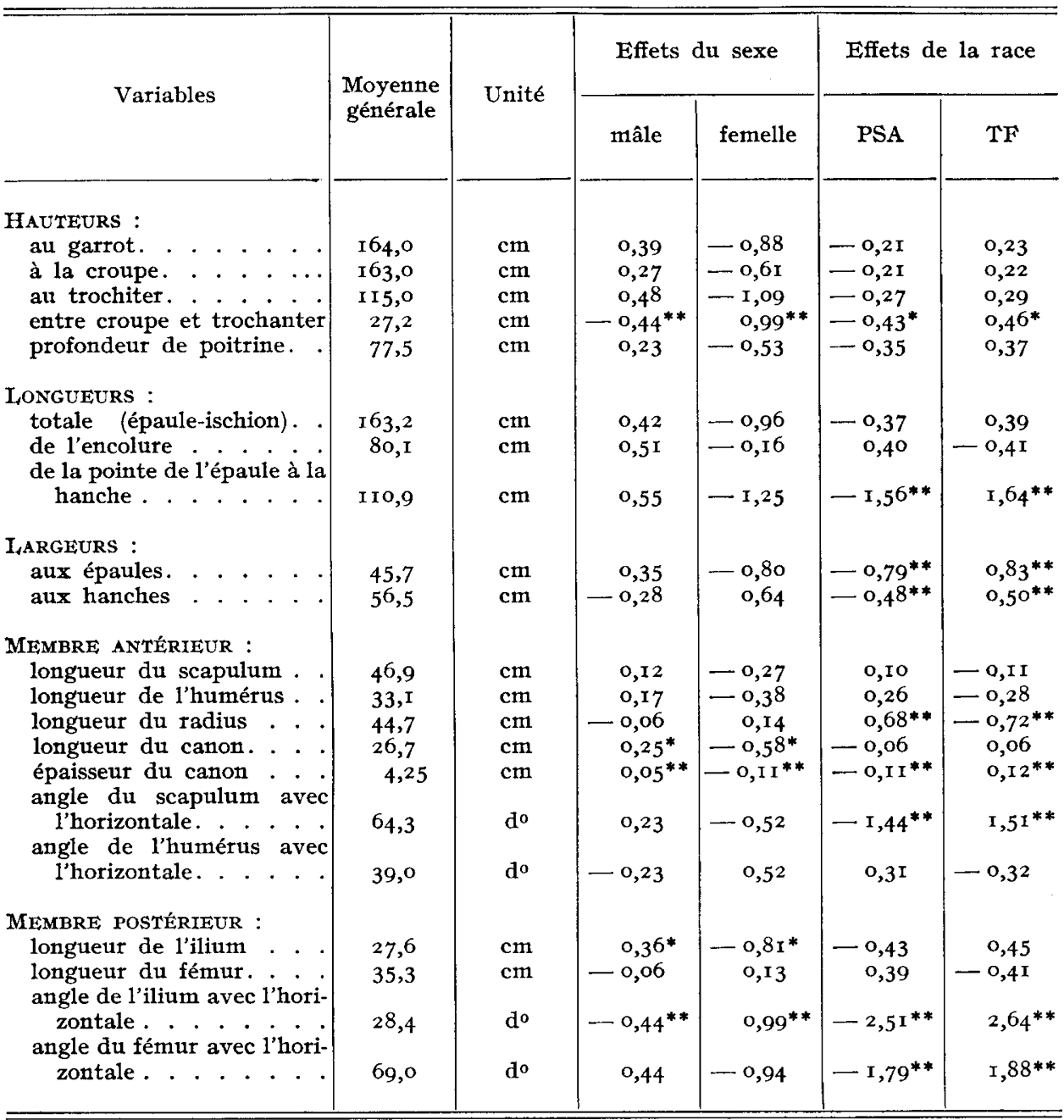

En ce qui concerne l'effet du sexe les deux analyses ont des résultats quelque peu différents. Dans la première (tabl. 2, 3 et 4) des effets significatifs se manifestent en faveur des mâles pour la longueur et 1'épaisseur du canon antérieur ainsi que pour la longueur de 1'ilium. En revanche la hauteur entre croupe et trochanter et l'angle de l'ilium avec l'horizontale apparaissent significativement plus forts chez les femelles. Dans la seconde analyse (tab1. 5 et 6 ) la supériorité des mâles s'affirme là où l'étude précédente ne marquait que des tendances. Il s'agit des hauteurs au garrot, au dos, à la croupe, à la pointe de la hanche, au trochanter, 
TABLEAU 4

Résultats de l'analyse de la variance:

estimées des effets d'interaction sexe-race significatifs sur les 40 trotteurs et les 42 Pur Sang

Analysis of variance:

estimates of significant sex-breed interactions on 40 trotters and 42 thoroughbreds

\begin{tabular}{|c|c|c|c|c|c|c|}
\hline \multirow{2}{*}{ Variables } & \multirow{2}{*}{$\begin{array}{l}\text { Moyenne } \\
\text { générale }\end{array}$} & \multirow{2}{*}{ Unité } & \multicolumn{2}{|c|}{ Pur Sang Anglais } & \multicolumn{2}{|c|}{ Trotteur Français } \\
\hline & & & mâle & femelle & mâle & femelle \\
\hline $\begin{array}{l}\text { MEMBRE ANTÉRIEUR : } \\
\text { longueur de } 1 \text { 'humérus } \\
\text { épaisseur du canon } .\end{array}$ & $\begin{array}{c}33, \mathrm{I} \\
4,25\end{array}$ & $\begin{array}{l}\mathrm{cm} \\
\mathrm{cm}\end{array}$ & $\begin{array}{l}-0,25 \\
-0,03\end{array}$ & $\begin{array}{l}0,8 \mathrm{I} \\
0,09\end{array}$ & $\begin{array}{l}0,32 \\
0,04\end{array}$ & $\begin{array}{l}-0,54 \\
-0,06\end{array}$ \\
\hline
\end{tabular}

du vide sous-sternal, de la longueur de l'encolure, de la largeur aux épaules, du périmètre thoracique, des longueurs du scapulum, de l'humérus, du radius, du fémur, du tibia et du canon postérieur ainsi que de l'épaisseur du canon antérieur.

\section{C. - Analyses en composantes principales}

De nombreuses analyses en composantes principales ont été conduites sur l'ensemble des échantillons mesurés. Avec quelques variantes elles confirment les deux seules que nous présentons, l'une (tabl. 7) parce qu'elle concerne un échantillon "tout venant " de chevaux de service, l'autre parce qu'elle touche un ensemble d'étalons sélectionnés, produisant des chevaux de sport (tab1. 8). Nous verrons ainsi que la structure des observations est très peu modifiée par la sélection. En effet, 1'examen des corrélations des variables de départ avec les 5 composantes principales de plus grande variance sont très comparables dans les deux analyses.

Le premier facteur qui représente respectivement 48 et 37 p. Ioo de la variance totale est en corrélation positive forte avec toutes les variables associées à la taille. "La taille" (hauteur au garrot) apparaît donc bien comme le premier facteur de tri envisageable pour classer des individus.

Le second facteur, indépendant du premier, exprime respectivement $\mathrm{r} 2$ et I 2 p. Ioo de la variance. Il traduit l'opposition des largeurs avec la hauteur du vide sous-sternal, c'est un facteur de "compacité ".

La troisième composante, indépendante des deux premières, exprime respectivement 6 et $8 \mathrm{p}$. Ioo de la variance totale. Elle fait apparaître 1'agencement et l'orientation des rayons postérieurs. Elle traduit aussi, bien que cela soit plus net dans la seconde analyse, l'opposition des angles de l'ilium et du fémur avec l'horizontale. Elle révèle donc une tendance à la constance de l'angle coxo-fémoral.

Le quatrième facteur (respectivement 6 et $7 \mathrm{p}$. Ioo de la variance totale) montre l'orientation et l'agencement des rayons antérieurs. De même que précédemment l'opposition des angles du scapulum et de l'humérus avec l'horizontale traduit la relative constance de l'angle scapulo-huméral. 


\section{TABLEAU 5}

Résultats de l'analyse de la variance:

signification des effets sexe et aptitude sur les 52 "Bons sauteurs" et les $5 \mathrm{I}$ "Mauvais"

Analysis of variance:

significance of sex and jumping ability effect on 52 "Good jumpers " and $5 I$ "Bad jumpcrs"

\begin{tabular}{|c|c|c|c|c|}
\hline \multirow{2}{*}{ Variables } & \multirow{2}{*}{$\begin{array}{l}\text { Carré } \\
\text { moyen } \\
\text { résiduel }\end{array}$} & \multicolumn{3}{|c|}{ Valeurs du " $F$ " } \\
\hline & & Interaction & $\begin{array}{l}\text { Effet du } \\
\text { sexe }\end{array}$ & $\begin{array}{l}\text { Effet de } \\
\text { l'aptitude }\end{array}$ \\
\hline Degrés de liberté . . . . . . . . & 99 & I & $\mathbf{I}$ & I \\
\hline $\begin{array}{l}\text { HAUTEURS : } \\
\text { au garrot. } \\
\text { au dos. }\end{array}$ & $\begin{array}{r}18,523 \\
24,268 \\
23,323 \\
21,246 \\
20,198 \\
9,216\end{array}$ & $\begin{array}{l}0,03 \\
1,62 \\
2,18 \\
0,75 \\
2,10 \\
2,47\end{array}$ & $\begin{array}{l}22,41^{* *} \\
\text { I } 8,14^{* *} \\
\text { I } 2,62^{* *} \\
\text { I } 6,67^{* *} \\
\text { I } 5,96^{* *} \\
\text { I } 2,33^{* *}\end{array}$ & $\begin{array}{l}2,83 \\
0,22 \\
1,59 \\
1,00 \\
1,21 \\
0,63\end{array}$ \\
\hline $\begin{array}{l}\text { LONGUEURS : } \\
\text { totale (épaule-ischion) . . . . . . . . . . . . . . } \\
\text { de l'encolure . } \\
\text { du haut du scapulum à la hanche. } \\
\text { du haut du scapulum à la rotule. }\end{array}$ & $\begin{array}{l}46,467 \\
26,356 \\
22,646 \\
29,815\end{array}$ & $\begin{array}{l}0,02 \\
0,27 \\
0,01 \\
0,07\end{array}$ & $\begin{array}{l}2,49 \\
\mathrm{I} \mathbf{I}, 97^{* *} \\
3,4 \mathrm{I} \\
\mathrm{I}, 29\end{array}$ & $\begin{array}{l}\mathrm{I}, 44 \\
0,97 \\
\mathrm{I}, 56 \\
\mathrm{I}, 62\end{array}$ \\
\hline $\begin{array}{l}\text { LARGEURS : } \\
\text { aux épaules }\end{array}$ & $\begin{array}{r}8,372 \\
11,498 \\
9,514 \\
52,532\end{array}$ & $\begin{array}{l}0,90 \\
0,28 \\
0,02 \\
0,18\end{array}$ & $\begin{array}{l}4,4 I^{*} \\
\mathrm{I}, 43 \\
0,84 \\
7,92^{* *}\end{array}$ & $\begin{array}{l}\text { г } 6,03^{* *} \\
5,49^{*} \\
\text { I } 2,92^{* *} \\
6,32^{*}\end{array}$ \\
\hline 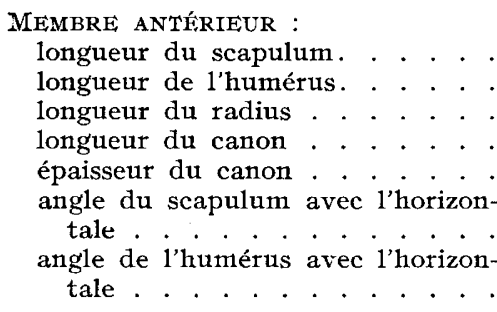 & $\begin{array}{r}6,146 \\
4,123 \\
4,040 \\
2,282 \\
8,319 \\
\\
\text { I2,566 } \\
\\
19,378\end{array}$ & $\begin{array}{l}1,62 \\
0,00 \\
2,16 \\
0,32 \\
0,63 \\
0,23 \\
\\
0,96\end{array}$ & $\begin{array}{c}4,24^{*} \\
3,92^{*} \\
6,09^{*} \\
1,25 \\
20,82^{* *} \\
1,5^{2} \\
0,25\end{array}$ & $\begin{array}{l}5,62^{*} \\
8,90^{* *} \\
0,04 \\
0,09 \\
0,48 \\
4,93^{*} \\
\\
2,39\end{array}$ \\
\hline 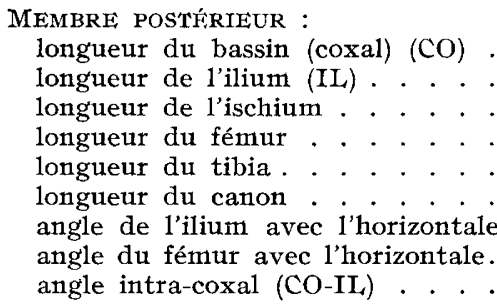 & $\begin{array}{r}\mathrm{r} 3,565 \\
4,063 \\
7,036 \\
4,460 \\
9, \mathrm{I} 74 \\
2,438 \\
38,899 \\
\text { I6,4 I } 5 \\
7, \mathrm{I} \text { I I }\end{array}$ & $\begin{array}{l}0,01 \\
0,02 \\
0,00 \\
0,19 \\
0,36 \\
1,52 \\
0,33 \\
0,48 \\
0,15\end{array}$ & $\begin{array}{l}0,57 \\
0,06 \\
0,99 \\
5,65^{*} \\
4,82^{*} \\
5,01^{*} \\
0,00 \\
3,75 \\
0,07\end{array}$ & $\begin{array}{l}2,72 \\
6,82^{*} \\
0,71 \\
2,25 \\
0,34 \\
2,62 \\
0,41 \\
9,40^{* *} \\
0,23\end{array}$ \\
\hline
\end{tabular}




\section{TABLEAU 6}

Résultats de l'analyse de la variance:

estimées des effets sexe et aptitude sur les 52 "Bons sauteurs " et les $5^{I}$ " Mauvais"

Analysis of variance:

estimation of sex and jumping ability effects on 52 "Good jumpers" and $5 I$ "Bad jumpers "

\begin{tabular}{|c|c|c|c|c|c|c|}
\hline \multirow{2}{*}{ Variables } & \multirow{2}{*}{$\begin{array}{l}\text { Moyenne } \\
\text { générale }\end{array}$} & \multirow{2}{*}{ Unité } & \multicolumn{2}{|c|}{ Effets du sexe } & \multicolumn{2}{|c|}{ Effets de l'aptitude } \\
\hline & & & mâle & femelle & "B" & "M⿻ \\
\hline \multicolumn{7}{|l|}{ HAUTEURS : } \\
\hline au garrot. . . . . . & 163,4 & $\mathrm{~cm}$ & $1,20^{* *}$ & $-3,37^{* *}$ & $0,7 \mathrm{I}$ & $-0,72$ \\
\hline au dos....... & 154,5 & $\mathrm{~cm}$ & $1,23^{* *}$ & $-3,47^{* *}$ & 0,23 & $-0,23$ \\
\hline à la croupe. & 162,3 & $\mathrm{~cm}$ & $\mathrm{I}, \mathrm{OI} * *$ & $-2,84^{* *}$ & 0,59 & $-0,6 \mathrm{I}$ \\
\hline à la pointe de la hanche. & 146,7 & $\mathbf{c m}$ & $\mathrm{I}, \mathrm{II} \mathrm{I}^{* *}$ & $-3,1 i^{* *}$ & 0,45 & $-0,46$ \\
\hline au trochanter. . . . . & I 34,4 & $\mathrm{~cm}$ & $1,05^{* *}$ & $-2,97^{* *}$ & $0,4^{8}$ & $-0,49$ \\
\hline du vide sous-sternal & $86, \mathrm{I}$ & $\mathrm{cm}$ & $0,63^{* *}$ & $-\mathrm{I}, 7^{* * *}$ & $-0,23$ & 0,24 \\
\hline \multicolumn{7}{|l|}{ LONGUEURS : } \\
\hline totale (épaule-ischion) & $\mathbf{I} 6 \mathrm{I}, 8$ & $\mathrm{~cm}$ & 0,63 & $-1,78$ & 0,80 & $-0,82$ \\
\hline $\begin{array}{l}\text { de l'encolure } \\
\text { du haut du scapulum à la }\end{array}$ & 79,0 & $\mathrm{~cm}$ & $\mathrm{I}, 04^{* *}$ & $-2,94^{* *}$ & 0,49 & $-0,5^{\circ}$ \\
\hline hanche & 90,3 & $\mathrm{~cm}$ & 0,52 & $-\mathrm{I}, 45$ & $-0,5^{8}$ & 0,59 \\
\hline totule . . . . . & $\mathrm{r} \times 6,3$ & $\mathrm{~cm}$ & 0,37 & - I,03 & $-0,68$ & 0,69 \\
\hline \multicolumn{7}{|l|}{ LARGEURS : } \\
\hline aux épaules. & 46,4 & $\mathrm{~cm}$ & $0,36^{*}$ & - I,OO* & $\mathrm{I}, \mathrm{I} 3^{* *}$ & $-\mathrm{I}, \mathrm{I} 5^{* *}$ \\
\hline aux hanches . & 57,5 & $\mathrm{~cm}$ & 0,24 & $-0,67$ & $0,78^{*}$ & $-0,79^{*}$ \\
\hline aux trochanters.... & 53,0 & $\mathrm{~cm}$ & $0, \mathrm{x} 7$ & $-0,47$ & $\mathrm{I}, 08^{* *}$ & 一 $\mathrm{I}, \mathrm{IO}^{* *}$ \\
\hline périmètre thoracique. . & I 88,3 & $\mathrm{~cm}$ & $1,20 * *$ & $-3,37^{* *}$ & $\mathrm{I}, 7^{*}$ & $-\mathrm{I}, 8 \mathrm{I}^{*}$ \\
\hline \multicolumn{7}{|l|}{ MEMBRE ANTÉRIEUR : } \\
\hline longueur $\mathrm{du}$ scapulum . & 52,0 & $\mathrm{~cm}$ & $0,30^{*}$ & - $0,84^{*}$ & $0,57^{*}$ & $-0,5^{8^{*}}$ \\
\hline longueur de l'humérus . & 33,8 & $\mathrm{~cm}$ & $0,24^{*}$ & $-0,67^{*}$ & $0,59^{* *}$ & $-0,60^{* *}$ \\
\hline longueur du radius . & 42,6 & $\mathrm{~cm}$ & $0,29^{*}$ & $-0,82^{*}$ & 0,04 & $-0,04$ \\
\hline longueur du canon. & 25,5 & $\mathrm{~cm}$ & 0, Io & $-0,28$ & 0,04 & $-0,04$ \\
\hline $\begin{array}{l}\text { épaisseur du canon } \\
\text { angle du scapulum avec }\end{array}$ & 46,2 & $\mathrm{~mm}$ & $0,77^{*}$ & $-2,18^{*}$ & 0,19 & $-0,20$ \\
\hline $\begin{array}{l}\text { l'horizontale. } \\
\text { angle de l'humérus avec }\end{array}$ & 68,4 & $\mathrm{gr}$ & 0,26 & $-0,72$ & $-0,77^{*}$ & $0,7^{*}$ \\
\hline l'horizontale. . . . & $44, \mathrm{I}$ & $g r$ & 0,13 & $-0,36$ & $-0,66$ & 0,68 \\
\hline \multicolumn{7}{|l|}{ MEMBRE POSTÉRIEUR : } \\
\hline $\begin{array}{c}\text { longueur du bassin (coxal) } \\
\text { (CO) }\end{array}$ & & $\mathrm{cm}$ & 0,16 & $-0,46$ & 0,59 & $-0,60$ \\
\hline longueur de 1'ilium (IL) & 27,7 & $\mathrm{~cm}$ & 0,03 & $-0,08$ & $0,5 I^{*}$ & $-0,5^{2 *}$ \\
\hline longueur de l'ischium . & $2 \mathbf{I}, 2$ & $\mathrm{~cm}$ & 0,16 & $-0,44$ & 0,22 & $-0,22$ \\
\hline longueur du fémur. . & $37, \mathbf{I}$ & $\mathrm{cm}$ & $0,29^{*}$ & $-0,83^{*}$ & $0,3 \mathrm{I}$ & $-0,32$ \\
\hline longueur du tibia. . & 48,3 & $\mathrm{~cm}$ & $0,39^{*}$ & - $\mathrm{I}, \mathrm{IO} \mathrm{O}^{*}$ & 0,17 & $-0,17$ \\
\hline $\begin{array}{l}\text { longueur du canon. } \\
\text { angle de l'ilium avec l'hori- }\end{array}$ & 35,4 & $\mathrm{~cm}$ & $0,2 I^{*}$ & $-0,5^{*}$ & 0,25 & $-0,25$ \\
\hline zontale . - - . - · & 29,6 & $\mathrm{gr}$ & $-0,02$ & 0,06 & $-0,39$ & $0,4^{\circ}$ \\
\hline 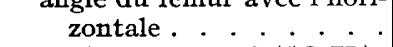 & 76,2 & $\mathrm{gr}$ & $-0,46$ & $\mathrm{I}, 3^{\circ}$ & $-I, 2 I * *$ & $I, 24^{*}$ \\
\hline angle intra-coxal (CO-IL). & I 2,8 & gr & 0,04 & $-0,12$ & $-0,13$ & 0,13 \\
\hline
\end{tabular}




\section{TABLEAU 7}

Coefficients de corrélation entre 30 variables

et les cinq composantes de plus grande variance sur un échantillon de 60 chevaux d'instruction

Correlation coefficients between 30 variables and the five main factors of component analysis made on 60 stock horses

\begin{tabular}{|c|c|c|c|c|c|}
\hline \multirow{2}{*}{ Variables } & \multicolumn{5}{|c|}{ Composantes } \\
\hline & Fact I & Fact 2 & Fact 3 & Fact 4 & Fact 5 \\
\hline \multicolumn{6}{|l|}{ HAUTEURS : } \\
\hline au garrot. . . . . . . . . . & 0,24 & 0,17 & $-0,00$ & 0,05 & 0,04 \\
\hline 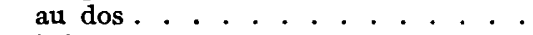 & 0,24 & 0,16 & 0,04 & 0,08 & 0,02 \\
\hline à la croupe. . . . . . . . . & 0,24 & 0,13 & $-0,00$ & 0,03 & $-0,02$ \\
\hline au sommet du scapulum. . . . . & 0,24 & 0,16 & 0,02 & 0,07 & 0,07 \\
\hline au trochiter. . . . . . . . & 0,23 & $0,2 \mathrm{I}$ & 0,09 & $-0,14$ & 0,05 \\
\hline au trochanter. . . . . . . . & 0,24 & 0,14 & $-0,06$ & $-0,00$ & 0,04 \\
\hline du vide sous-sternal. . . . . . & 0,17 & 0,34 & 0,07 & 0,14 & $-0, \mathrm{II}$ \\
\hline \multicolumn{6}{|l|}{ LONGUEURS : } \\
\hline totale (épaule-ischion) & 0,23 & $-0,10$ & 0,03 & $-0,03$ & $-0,15$ \\
\hline de l'encolure . . . . . . . . & 0,15 & 0,15 & 0,09 & $0,3 \mathrm{I}$ & 0,03 \\
\hline $\begin{array}{l}\text { de la pointe de l'épaule à la hanche. } \\
\text { de la pointe de l'épaule à la dernière }\end{array}$ & 0,17 & $-0,14$ & 0,16 & $-0,03$ & $-0,36$ \\
\hline côte . . . . . . . . & $0, \mathrm{I} 8$ & $-0,17$ & 0,14 & o,ro & $-0,20$ \\
\hline \multicolumn{6}{|l|}{ LARGEURS： } \\
\hline aux épaules. & 0,15 & $-0,3 \mathrm{I}$ & $-0, \mathbf{1} 2$ & $-0,00$ & 0,06 \\
\hline aux hanches. . & 0,19 & $-0,23$ & 0,15 & 0,12 & $-0,01$ \\
\hline aux trochanters. . . . & 0,16 & $-0,33$ & -0, II & 0,08 & $0, x \mathrm{I}$ \\
\hline périmetre thoracique. . . . . . & 0,20 & $-0,22$ & 0,03 & $-0,15$ & 0,17 \\
\hline périmètre scapulo-thoracique . . . & $0,2 \mathrm{I}$ & $-0,28$ & $-0,0 \mathrm{I}$ & $-0,09$ & 0,19 \\
\hline \multicolumn{6}{|l|}{ MEMBRE ANTERIEUR : } \\
\hline lonģueur $\mathrm{du}$ scapulum. . . . . . . & 0,17 & $-0,14$ & $-0,25$ & 0,16 & 0,10 \\
\hline longueur de l'humérus. . . . . . & 0,16 & $-0,00$ & $-0,05$ & $-0,25$ & $-0,22$ \\
\hline longueur du radius . . . . . . . . & 0,19 & 0,26 & $-0,07$ & $-0,08$ & 0,10 \\
\hline longueur du canon ....... & 0,19 & 0,18 & $-0,04$ & $-0,08$ & 0,07 \\
\hline épaisseur du canon ....... & 0,17 & $-0,24$ & 0,24 & $0, \mathrm{I} 8$ & $0, \mathbf{I} 6$ \\
\hline angle $\mathrm{du}$ scapulum avec l'horizontale & $-0,00$ & 0,10 & 0,04 & 0,65 & 0,05 \\
\hline angle de l'humérus avec l'horizontale & 0,03 & 0,10 & 0,35 & $-0,42$ & 0,18 \\
\hline \multicolumn{6}{|l|}{ MEMBRE POSTÉRIEUR : } \\
\hline longueur $\mathrm{du}$ bassin (coxal). . . . . & 0,15 & $-0,14$ & $-0,16$ & $-0,06$ & $-0,28$ \\
\hline longueur du fémur . . . . . . & 0,17 & 0,02 & $-0,37$ & $-0,06$ & $-0,12$ \\
\hline longueur du tibia . . . . . . & 0,19 & 0,07 & 0,19 & $-0,07$ & $-0, \mathrm{II}$ \\
\hline longueur $\mathrm{du}$ canon $\ldots \ldots$ & 0,18 & 0,13 & $-0,23$ & $-0,15$ & $-0,08$ \\
\hline épaisseur du jarret $\ldots$ & 0,19 & $-0,19$ & 0,23 & 0,00 & 0,04 \\
\hline angle de l'ilium avec l'horizontale. . & $-0,02$ & $-0,03$ & 0,58 & 0,02 & 0,00 \\
\hline angle du fémur avec l'horizontale. . & 0,04 & $-0,03$ & $-0, \mathrm{II}$ & $-0,09$ & 0,68 \\
\hline Pourcentage cumulé de la variance. & 0,49 & $0,6 \mathbf{I}$ & 0,67 & 0,73 & 0,77 \\
\hline
\end{tabular}




\section{TABLEAU 8}

Coefficients de corrélation entre $3 I$ variables

et les cinq composantes de plus grande variance sur un échantillon de 94 étalons nationaux

Correlation coefficients between $3 I$ variables

and the five main factors of component analysis made on 94 selected stallions of national studs

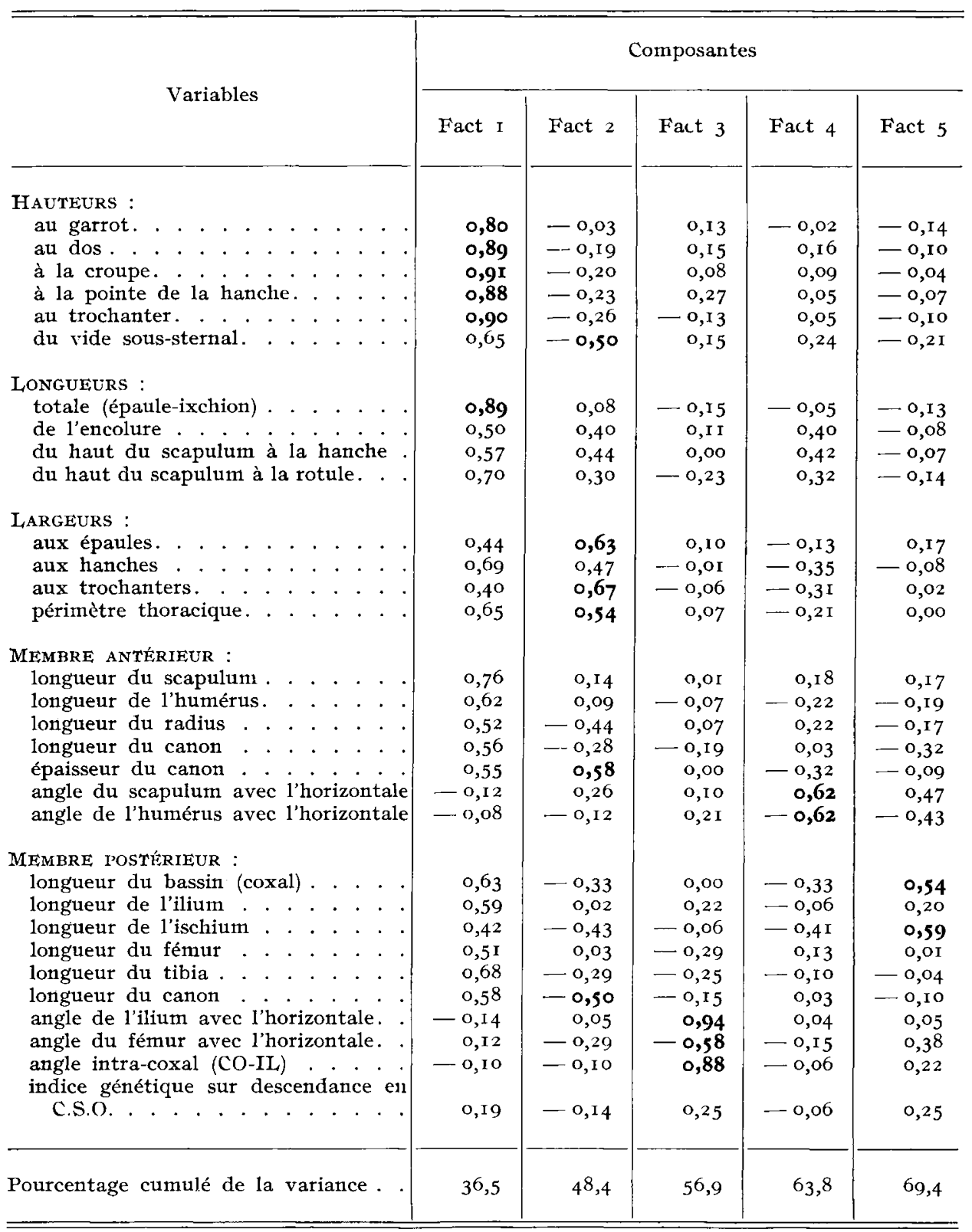


Les composantes suivantes représentent des fractions toujours plus faibles de la variance, nous ne les commentons pas ici. En effet la prise en compte des facteurs " taille " et " compacité " traduisant de 1'ordre de la moitié de la variance totale paraît donc le plus souvent suffisante pour classer les animaux. La représentation des 94 étalons mesurés, d'après leur valeur pour les facteurs I et 2 (" taille" et " compacité "), reportée figure 5 , en est une bonne illustration :

- les étalons de Selle Français, regroupés dans la partie supérieure et à droite, présentent une plus grande taille et une plus forte compacité;

- les étalons Pur Sang Anglais centrés dans le demi plan inférieur sont caractérisés par une taille moyenne et homogène, et une faible compacité;

- les étalons Anglo-Arabes occupant la partie intermédiaire, se divisent en deux sous-populations. Un premier noyau situé juste entre les Pur Sang Anglais et les Selle Français regroupe les Anglo-Arabes très proches du Pur Sang Anglais. Le reste se plaçant en haut et à gauche du plan rassemble les individus petits et compacts, proches morphologiquement du cheval Arabe.

Les 3 étalons Trotteur Français sont trop peu nombreux pour que 1'on puisse conclure.

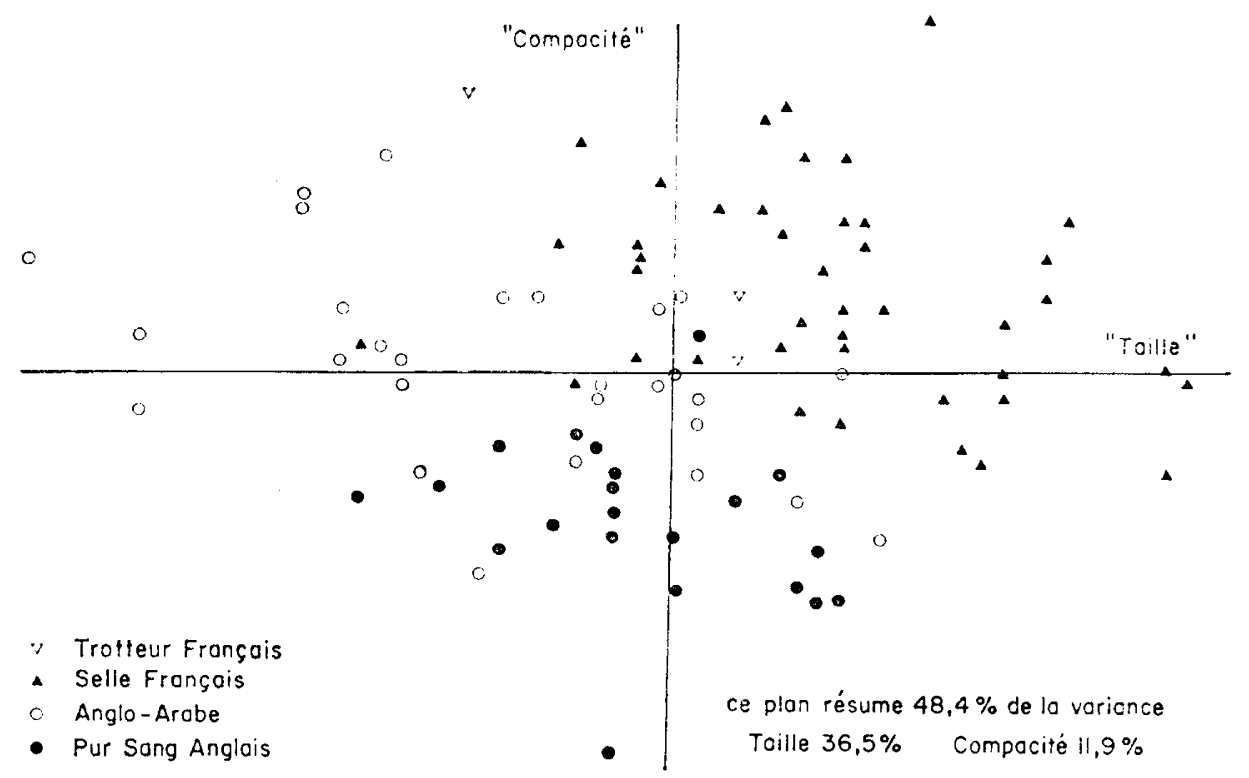

FIG. 5. - Situation des 94 étalons des différentes races en projection sur un plan déterminé par les deux premières composantes principales " taille "et "compacité ". Position of the 94 stallions of different breeds on the plane

defined by the two main factors of the component analysis " herght " and "compactness"

\section{D. - Analvses discriminantes à deux populations}

Nous ne présentons ici que les fonctions discriminantes obtenues au seuil de 5 p. roo. 
Le tableau 9 donne les résultats de cette analyse pour les Pur Sang Anglais et les Trotteur Français. La figure 6 retrace par race les histogrammes de répartition des chevaux en fonction de la probabilité d'être classés a posteriori parmi les Trotteurs.

\section{TABLEAU 9}

Résultats de l'analyse discriminante au seuil de 5 p. Ioo appliquée à 40 trotteurs et 42 Pur Sang sur les variables corrigées pour l'effet sexe et celui des interactions race-sexe

Results of discriminant analysis at the 5 p. Ioo level applied to 40 trotters and 42 thoroughbreds on variables corrected for sex and sex-breed interaction effects

\begin{tabular}{|c|c|c|c|}
\hline $\begin{array}{c}\text { Variables introduites } \\
\text { (dans l'ordre) }\end{array}$ & Unité & $\begin{array}{l}\text { Valeurs } \\
\text { successives } \\
\text { du } D^{2}\end{array}$ & $\begin{array}{l}\text { Coefficient } \\
\text { de la fonction } \\
\text { discriminante }\end{array}$ \\
\hline 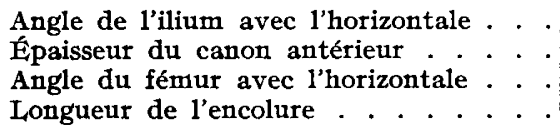 & $\begin{array}{l}d^{\circ} \\
\mathrm{cm} \\
\mathrm{d}^{0} \\
\mathrm{~cm}\end{array}$ & $\begin{array}{r}4,4^{8} \\
8,40 \\
9,75 \\
10,61\end{array}$ & $\begin{array}{r}0,075 \\
0,792 \\
0,021 \\
-0,013\end{array}$ \\
\hline Terme constant. . . . . & & & $-5,885$ \\
\hline
\end{tabular}

\section{TABLEAU IO}

Résultats de l'analyse discriminante au seuil de 5 p. 100 appliquée à $5^{2}$ "Bons sauteurs " et $5^{I}$ " Mauvais "

Results of discriminant analysis at the 5 p. roo level applied to 52 "Good jumpers " and 51 "Bad jumpers "

\begin{tabular}{|c|c|c|c|}
\hline $\begin{array}{l}\text { Variables introduites } \\
\text { (dans l'ordre) }\end{array}$ & Unité & $\begin{array}{c}\text { Valeurs } \\
\text { successives } \\
\text { du } D^{2}\end{array}$ & $\begin{array}{l}\text { Coefficient } \\
\text { de la fonction } \\
\text { discriminante }\end{array}$ \\
\hline 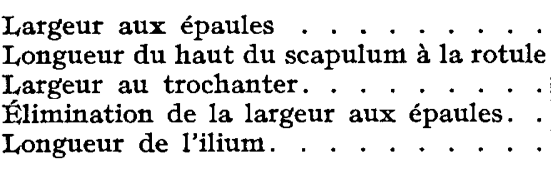 & $\begin{array}{l}\mathrm{cm} \\
\mathrm{cm} \\
\mathrm{cm} \\
\mathrm{cm}\end{array}$ & $\begin{array}{l}0,55 \\
0,79 \\
I, 26 \\
I, 2 I \\
I, 5 I\end{array}$ & $\begin{array}{l}-\overline{0,043} \\
\overline{0,074} \\
\overline{0,056}\end{array}$ \\
\hline Terme constant. . . . . . . . . . . . & & & $-0,465$ \\
\hline
\end{tabular}


Le tableau ro et la figure 7 rendent compte des mêmes résultats dans le cas des "Bons" et des "Mauvais sauteurs".

On s'aperçoit pour le premier échantillon qu'au seuil de 5 p. 1004 variables, l'angle de l'ilium avec 1'horizontale, celui du fémur, l'épaisseur du canon et la longueur de l'encolure, contribuent significativement à une discrimination qui s'avère satisfaisante puisque sur les 82 chevaux seuls deux galopeurs sont classés parmi les trotteurs et inversement deux trotteurs parmi les Pur Sang. Le pourcentage de déclassés n'est donc que de $5 \mathrm{p}$. Ioo.

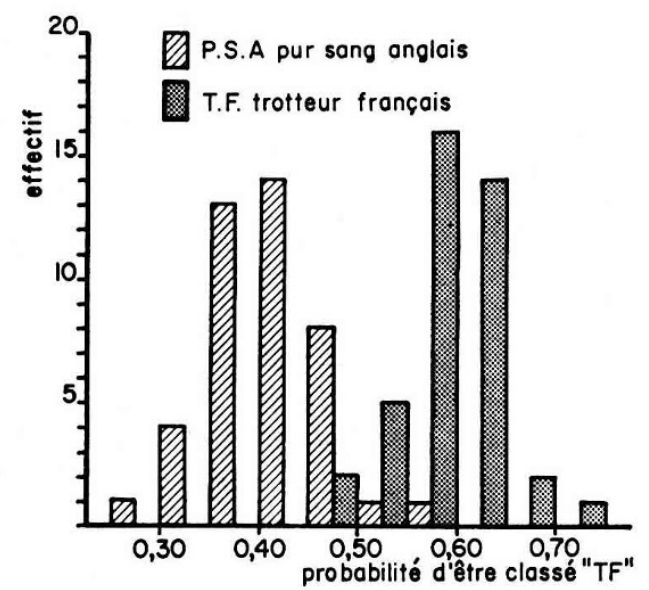

FIG. 6. - Histogrammes par race des chevaux en fonction de leur probabilité d'être classés a posteriori parmi les Trotteurs Français.

Distribution of the horses per breed according to their probability of being ranked among the French Trotters.

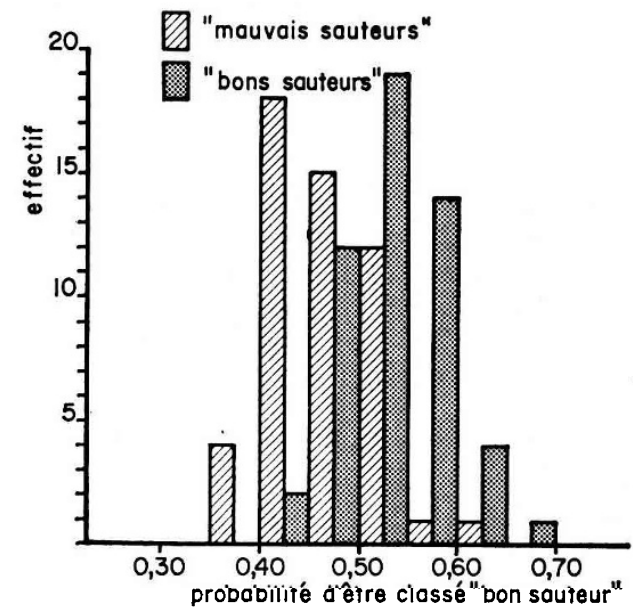

FIG. 7. - Histogrammes par groupe des chevaux en fonction de leur probabilité d'être classés a posteriori parmi les "Bons sauteurs".

Distribution of the horses per group according to their probability of being ranked among the * good jumpers 》. 
Pour le second échantillon au seuil de $5 \mathrm{p}$. roo 3 variables seulement sont retenues dans l'équation : la largeur au trochanter, la longueur du sommet du scapulum à la rotule et la longueur de l'ilium. Mais dans ce cas 27 p. Ioo des chevaux sont déclassés. Le pouvoir discriminant des mensurations apparaît donc beaucoup plus faible que précédemment.

\section{E. - Régressions multiples progressives}

\section{TABLEAU II}

Coefficients des équations de régression multiple de I5 variables morphologiques sur l'indice génétique de 45 étalons de Selle Français $(S F), 28$ Anglo-arabes $(A A)$

et 21 Pur Sang Anglais $(P S A)$

- Les équations sont celles qui présentent un maximum de coefficients significativement différents de $o$. Elles correspondent à l'arrivée au palier de détermination.

- Les coefficients sont les "beta " de l'équation lorsque les variables sont standardisées.

Coefficients of the polynomial regression predicting, with 15 variables

the progeny testing index of different stallions: 45 French Saddle Breds $(S F), 28$ Anglo arabians $(A A)$ and $2 x$ Thoroughbreds $(P S A)$

- Equations presented are those having a maximum of significant coefficients. They correspond to the beginning of the determination plateau.

- All variables were standardized.

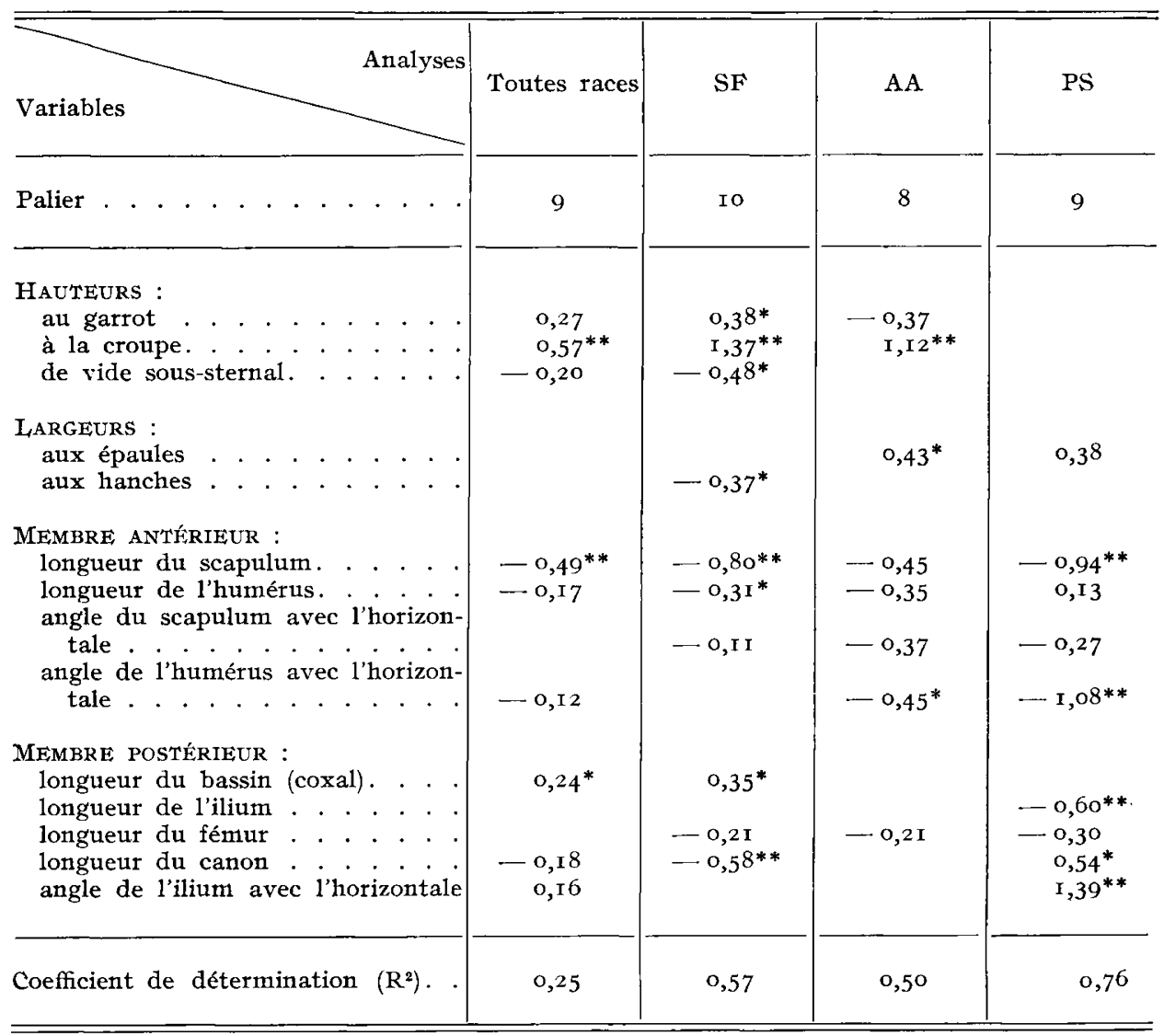


Lorsque 1'on cherche une équation linéaire de prédiction et que 1'on n'introduit successivement que des variables apportant une contribution significative à la détermination, les résultats des analyses apparaissent peu satisfaisants. Pour l'ensemble des 94 étalons l'indice génétique I peut être estimé par l'équation suivante :

$$
\mathrm{I}=-272,5+2,2 \mathrm{HG}
$$

$$
\left(\mathrm{R}^{2}=0,06\right)
$$

$\mathrm{HG}$ représentant la hauteur au garrot en centimères, $\mathrm{R}^{2}$ est le coefficient de détermination de la régression. Intra-race bien que légèrement plus précis, les modèles n'apportent guère plus de renseignements.

$\mathrm{I}=-49 \mathrm{I}, \mathrm{I}+3,5 \mathrm{HG}$, pour les 45 étalons de Selle Français $\left(\mathrm{R}^{2}=0, \mathrm{IO}\right)$.

$\mathrm{I}=206,2-2,9 \mathrm{AH}, \mathrm{AH}$ étant l'angle de 1'humérus avec 1'horizontale en grades pour les $2 S$ étalons $A$ nglo-Arabes $\left(\mathrm{R}^{2}=0, \mathrm{I}_{5}\right)$.

$\mathrm{I}=32, \mathrm{I}+2,7 \mathrm{AI}$, AI étant l'angle de l'ilium avec l'horizontale en grades pour les 2 I étalons $P$ ur Sang Anglais $\left(\mathrm{R}^{2}=0,24\right)$.

L'effectif de notre échantillon ne nous permet donc pas de fournir des équations de prédiction de l'indice génétique par les mensurations corporelles qui soient véritablement satisfaisantes. Il permet en revanche d'analyser des tendances.

\section{TABLEAU I2}

Moyennes des "Bons " et " Mauvais sauteurs", des étalons Selle Français, Anglo-arabes et Pur Sang Anglais pour les variables significatives dans les analyses

Average values of the "Good" and "Bad jumpers", and of the stallions of different breeds French Saddle, Anglo-arabian and Thoroughbred for the most important variables in the analyses

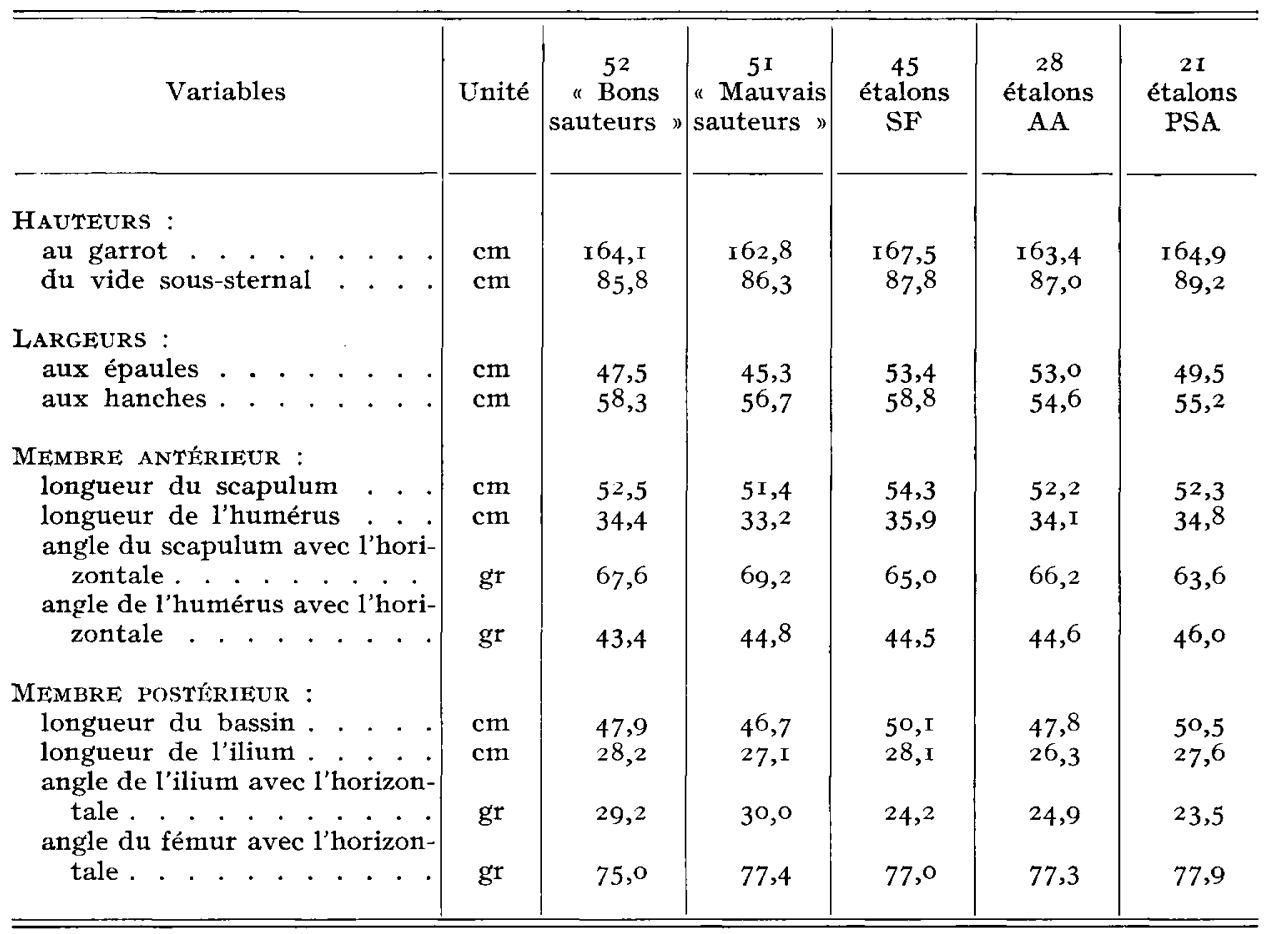




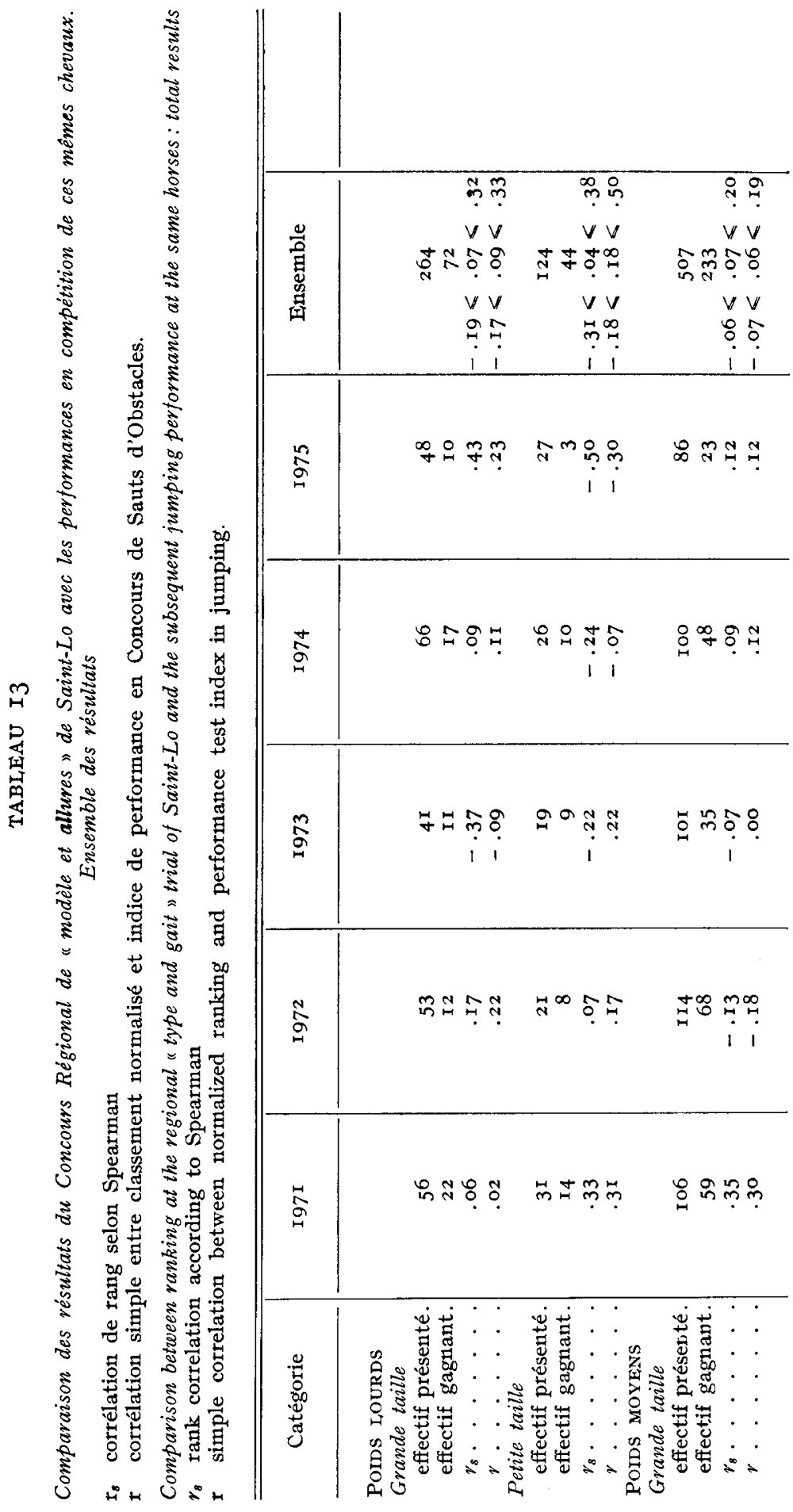




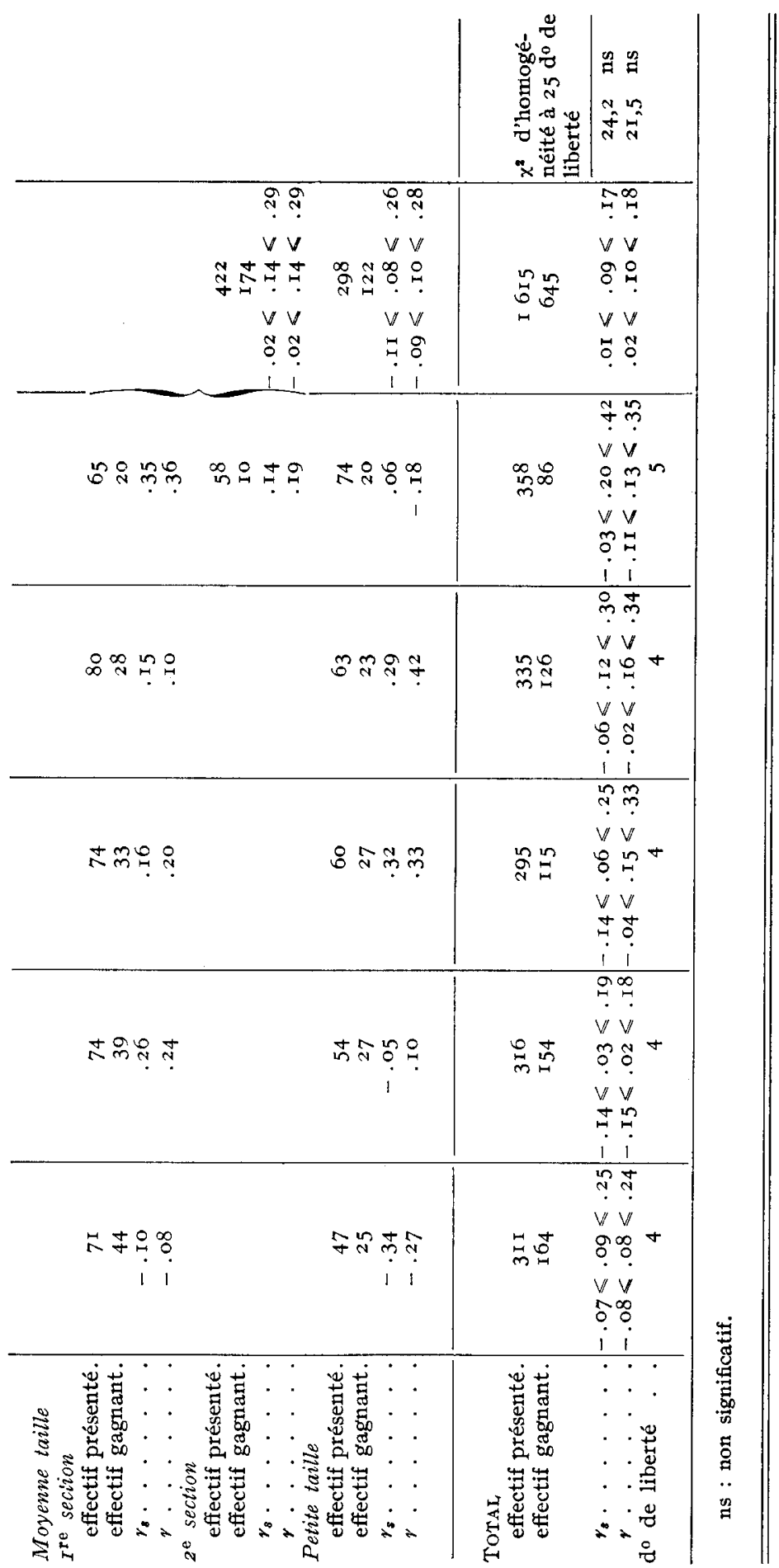


Ainsi l'examen du signe des coefficients significativement différents de zéro dans les équations de régression reportées (tabl. II) permet de mieux éclairer la conformation désirable chez un étalon destiné à produire des chevaux d'obstacles. Dans l'ensemble les résultats convergent avec l'analyse précédente, toutefois quelques contradictions apparaissent notamment au niveau de la longueur du scapulum, de celle de l'humérus, de l'angle du scapulum avec l'horizontale L'examen des moyennes des différents échantillons (tabl. I2) permet cependant de les résoudre en partie.

\section{F. - " Modèle et allures" et performances en Concours de Sauts d'Obstacles}

Le tableau I 3 fourn:t les résultats détaillés de l'étude des concours régionaux de " modèle et allures " de Saint--Lo. La valeur du " chi 2 " du test d'homogénéité des 26 corrélations correspondantes aux 26 classements analysés est de 24,2 pour les corrélations de rang $\left(r_{s}\right)$ et de $2 \mathrm{I}, 5$ pour les corrélations simples $(r)$. Elles ont respectivement les probabilités $0,5 \mathrm{r}$ et 0,66 d'être dépassées. Nous sommes donc fondés dans chacun des cas à établir des moyennes. Par les deux méthodes, les corrélations entre le jugement " modèle et allures " et l'indice de sélection sur performance individuelle sont très voisines

$$
r_{s}=0,09 \text { et } r=0, \text { ro }
$$

\section{III. - Discussion}

\section{A. - Validité de la méthode de mensuration}

Les valeurs de répétabilité obtenues (tabl. I) montrent que la méthode photométrique est utilisable en routine pour étudier des populations et caractériser certaines variations ou différences. En revanche à l'échelon individuel, on obtient généralement plus de précision en mesurant avec soin par les instruments classiques. Le temps nécessaire est toutefois incomparablement plus long. La méthode photographique s'impose donc à chaque fois que l'on désire relever un grand nombre de mensurations sur des effectifs relativement importants. Cependant pour des études plus limitées le mètre, la toise ou le ruban métrique paraissent plus adaptés. Pour la mesure des angles elle s'avère toutefois le plus souvent préférable, puisque les méthodes classiques ne dispensent pas du repérage et qu'en outre elles ne permettent pas d'obtenir la simultanéité des estimations que donne la photographie. En effet selon la position du cheval les angles articulaires peuvent varier sensiblement.

Les exigences de la méthode sur lesquelles on se doit d'insister sont :

- le repérage précis du "mètre étalon " dont les extrémités doivent être visibles très facilement quel que soit le fond et qui doit être suspendu verticalement dans le plan des mesures;

- le placé " standard " des points de repère; habitude qui s'acquiert facilement au cours d'essais préliminaires;

- le positionnement d'aplomb sur les quatre membres du cheval, sur une aire plane goudronnée ou bétonnée, pour permettre la matérialisation de 1'horizontale. Cette dernière exigence est de loin celle qui demande le plus d'attention. 
Par ailleurs si toutes les mensurations réalisées dans cette étude ne sont pas toujours nécessaires, les analyses en composantes principales nous montrent qu'un choix de variables doit au minimum rendre compte des quatre facteurs mis en évidence. Ce sont dans l'ordre :

- la taille (hauteur au garrot);

- la compacité (rapport d'une largeur au vide sous-sterna1);

- l'architecture des rayons osseux postérieurs (angle de l'ilium et du fémur avec 1'horizontale);

- l'orientation et les dimensions des rayons antérieurs (angle du scapulum et de l'humérus avec l'horizontale).

Des différenciations plus fines, hauteur de la croupe par rapport au garrot, longueur totale par rapport à ce même garrot apparaissent, nous le voyons, très secondaires pour différencier les individus des échantillons mesurés. En effet la variation de la taille et du format prime très largement sur le fait que les chevaux soient construits en " montant ", " en descendant " ou " plus long que haut "; cet aspect constituant un second niveau d'analyse qui n'a pas encore été abordé.

\section{B. - L'effet du sexe}

Les tableaux 3 et 6 donnent les différences entre sexe. Les femelles, de format (" taille et compacité ") plus réduit, semblent avoir le bassin relativement plus large que les mâles. En effet la supériorité de ceux-ci pour la largeur aux épaules et le périmètre thoracique n'apparaît plus significative pour la largeur aux hanches et aux trochanters (tabl. 6). Ceci se trouve confirmé par les tendances observées (tabl. 3) dans les deux races de course. En revanche si les femelles de Pur Sang Anglais et de Trotteur Français ont le bassin plus incliné, confirmant ainsi les résultats de BÉNARD (I968), aucune différence n'apparaît dans l'orientation des rayons pour les chevaux de Selle.

\section{C. - Les conséquences de la sélection sur performance chez le Trotteur Français et le Pur Sang Anglais}

Au début du siècle GASTE (I908) constatait déjà des divergences entre ces deux races caractérisées par une épaule plus droite et une croupe plus inclinée chez les Trotteurs. Nous confirmons pour notre part ces résultats qui furent longtemps débattus.

Pour le membre postérieur, l'angle de l'ilium avec l'horizontale a été trouvé de 5 degrés plus élevé chez les trotteurs. Le fémur en revanche ne bascule pas vers l'avant du fait de cette inclinaison puisque son angle avec l'horizontale est de 3,7 degrés supérieur chez les trotteurs. Pour le membre antérieur, les différences portent sur l'angle du scapulum avec l'horizontale qui est plus élevé de 2,9 degrés chez les trotteurs, le radius qui est plus court et l'épaisseur du canon qui est très notablement supérieure. En outre de taille comparable aux Pur Sang Anglais, les Trotteurs Français sont plus larges et donc sensiblement plus compacts.

Nous reportons le lecteur au travail de LEGAULT (1977) pour l'interprétation anatomodynamique de ces divergences qui traduisent dans chacune des deux races une adaptation fonctionnelle au trot ou au galop. En effet au trot comme au galop, la vitesse ne dépend pas tant du rythme des foulées que de l'étendue couverte 
à chaque battue (DUSE $\mathrm{k}$ et al., I970). Pour aller vite un cheval doit donc chercher le terrain loin devant lui; au trot sans qu'au moins un des bipèdes diagonaux ne quitte le sol; au galop par bonds. Le problème se trouve donc résolu de deux façons :

- chez le trotteur par flexion latérale du rachis et du bassin accompagnée d'un engagement maximum du fémur grâce à la position inclinée du coxal. Ce mouvement est le seul à permettre au postérieur de s'engager à l'extérieur du pied antérieur qui lui correspond et donc d'aller trouver le sol le plus loin possible sans gêner l'évolution de ce dernier. La grande ouverture naturelle de l'angle coxo-fémoral permet une phase d'appui et donc de propulsion prolongée;

- au galop, l'engagement des postérieurs n'est pas gêné par la position des antérieurs. Il se réalise alors par une flexion verticale du rachis qui fait basculer le coxal et par la fermeture de 1'angle coxo-fémoral. La phase d'appui et de propulsion est prolongée du fait de la position naturelle plus horizontale des os du bassin;

- à l'avant, les problèmes sont comparables si ce n'est que le Pur Sang bondissant doit aller poser les pieds le plus loin possible (scapulum incliné, radius long) alors que le Trotteur confronté au même impératif doit en plus dégager son antérieur après l'appui le plus rapidement possible (radius plus court entraînant une moindre inertie, épaule légèrement plus droite).

Les résultats des allemands NEISSER (I976), Scheide (I968), BURGER (I97r) qui concluaient à la nécessité d'une grande taille et d'une faible compacité pour le galopeur et d'une petite taille et d'une forte compacité pour le trotteur, ne se trouvent confirmés que pour le second aspect. L'absence d'effets sur la taille est peut-être due à 1'importance des courses " au monté » en France. De l'avis général cela confère au Trotteur Français son format important qui constitue son originalité principale par rapport aux autres races de Trotteurs dans le monde.

\section{D. - Le modèle du sauteur}

Chez les étalons, la taille paraît déterminer une bonne part de l'aptitude, elle ne varie en revanche pratiquement pas entre "Bons " et "Mauvais sauteurs". I1 semble que l'on doive distinguer aptitude aux gains en épreuves et aptitude au saut proprement dite. En effet même si un petit cheval ou un poney peut sauter relativement haut il est limité par sa taille. En compétitions officielles, les dotations augmentent fortement avec la classe de l'épreuve qui est fonction de la hauteur des obstacles. Il est donc logique que le facteur taille intervienne au niveau de l'explication de l'indice calculé par les gains où une notion de puissance s'ajoute à la notion d'aptitude au saut et qu'en revanche il n'apparaisse pas au niveau de 1'aptitude à l'obstacle jugée sans critères quantitatifs.

Toutes les analyses montrent la nécessité pour un sauteur d'être compact. Ce facteur reflète le potentiel de développement des masses musculaires soit la valeur athlétique du cheval et aussi une certaine aptitude à l'équilibre découlant d'un centre de gravité bas associé à un large polygone de sustentation.

L'opposition des angles de l'ilium et du fémur avec l'horizontale se traduit chez le sauteur par le basculement du coxal qui entraîne ainsi l'engagement du fémur sous la masse. D'autre part, la croupe est longue et large mais les hanches ne semblent pas devoir être trop saillantes car dans les régressions, les coefficients affectés à la largeur aux hanches sont négatifs. 
Pour le membre antérieur il semble que les "Bons sauteurs " aient à la fois un scapulum et un humérus plus proches de l'horizontale que ne l'ont les "Mauvais ". La fermeture de l'angle scapulo-huméral malgré sa relative constance paraît donc souhaitable. Les divergences rencontrées dans l'étude pour les longueurs du scapulum et de l'humérus se résolvent en considérant les moyennes des différents échantillons (tabl. I2) par la présence probable d'optimums (de l'ordre de $52 \mathrm{~cm}$ pour le scapulum et vers $34 \mathrm{~cm}$ pour l'humérus).

En résumé le sauteur se caractérise par une taille plutôt grande, une forte compacité et une croupe bien inclinée, longue, large et rectangulaire vue du dessus. L'angle du scapulum avec l'horizontale est relativement faible, celui de l'humérus et celui du fémur étant plutôt fermés. Ce cheval paraît avoir une prédisposition naturelle du rassemblé. Ces résultats sont en accord avec ceux de Schwark et al. I 977 et de FABIANI de I972 à I975. Toutefois ces caractéristiques morphologiques distinguant le sauteur sont beaucoup plus fines que celles permettant de différencier un trotteur d'un galopeur. Le pourcentage élevé de chevaux déclassés dans l'analyse discriminante en témoigne. Les résultats des corrélations entre les classements sur " modèle et allures " et performances ultérieures en compétition, faibles, en sont également l'indication. Signalons en outre que la grande variabilité morphologique des chevaux de selle ajoute à la confusion. Pour une race plus homogène telle que le Pur Sang Anglais, les données du problème sans être évidentes paraissent néanmoins plus simples. Une croupe bien inclinée, un humérus relativement horizontal, un scapulum pas trop long semblent en effet de bons indicateurs pour le choix des pur sang de croisement.

\section{E. - Morphologie et amélioration génétique}

L'étude des corrélations entre des variables morphologiques et des indices de sélection nous permet d'envisager l'estimation de l'efficacité relative de plusieurs méthodes pour l'amélioration génétique de l'aptitude aux sauts d'obstacles.

\section{TABLEAU I4}

Corrélations $(v)$ entre Indice génétique en "C.S.O. " $\left(X_{1}\right)$, morphologie $\left(X_{2}\right)$ et valeur génétique en "C.S.O. " $(Y)$

Correlations $(r)$ between genetic indexes in jumping $\left(X_{1}\right)$, conformation $\left(X_{2}\right)$ and breeding value for jumping ability $(Y)$

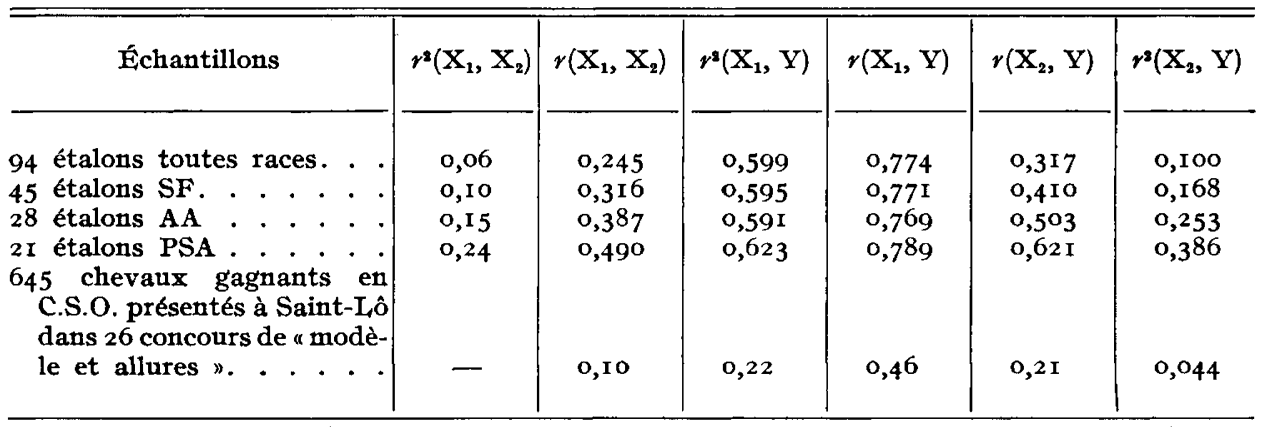

C.S.O. est l'abréviation de Concours de Sauts d'Obstacles.

$r^{2}$ carré des corrélations, exprime le pourcentage de détermination d'une variable par l'autre. 
En effet, conformément à ce qui a été exposé plus haut sous le titre de méthodes statistiques et génétiques, on peut établir les corrélations entre différents critères de sélection et la valeur génétique en Concours de Sauts d'Obstacles d'après nos résultats (tab1. I4). Le progrès génétique étant proportionnel à cette corrélation on peut donc classer les différents critères par ordre d'efficacité relative (tabl. I5).

TABLEAU I 5

Efficacité relative de différentes méthodes de sélection en fonction de leur précision ( $\mathrm{I}$ )

Relative efficiency of different selection methods in terms of accuracy

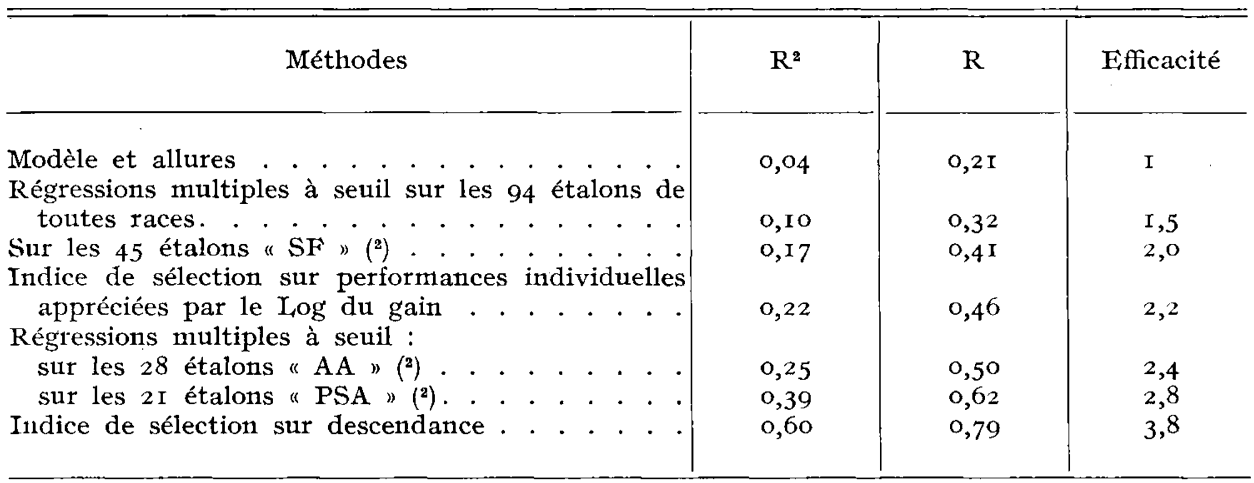

(1) Voir l'origine des données au tableau $\mathrm{I}_{3}$.

(2) Ces résultats sont fournis sous toute réserve étant donné les effectifs.

Nous constatons que si nous prenons comme référence l'efficacité de la méthode actuelle de sélection sur " modèle et allures ", le choix des reproducteurs sur un indice de performances individuelles établi sur la base des gains est environ deux fois plus efficace. Un indice de sélection d'étalon sur descendance établi aussi d'après les gains l'est environ quatre fois. Par ailleurs, les mensurations corporelles traitées de façon statistique apparaissent préférables au jugement à 1'œil nu. Globalement elles sont cependant moins précises que 1'indice individuel sur performance. Toutefois, malgré les réserves formulées en raison des faibles effectifs, l'élaboration de modèles intra-raciaux semble permettre d'égaler ou même de surpasser l'information fournie par les gains. Ceci demanderait cependant à être confirmé sur de plus larges échantillons.

\section{Conclusion}

Nous avons pu montrer par l'utilisation d'une méthode photométrique comment la sélection sur performances avait chez le Pur Sang Anglais et le Trotteur Français fixé des modèles différents de plus en plus adaptés aux allures rapides au galop comme au trot. Ces divergences mises en évidence et caractérisées surtout sur l'avant-main par GASTE dès rgo2 semblent plus accentuées de nos jours sur l'arrière-main. 
A 1'opposé, dans le cas de 1'aptitude aux sauts d'obstacles, il semble que le choix au modèle ne soit pas en mesure de faire progresser très rapidement 1'aptitude moyenne des chevaux pour plusieurs raisons. D'une part, on ignore le plus souvent le type de cheval qui est le plus adapté, d'autre part les critères de conformation ne sont pas les seuls à intervenir dans la réalisation de l'aptitude.

Cette étude a tenté de répondre a.u premier point et sans fournir de réponses très précises elle permet néanmoins de tracer les grandes lignes de la conformation du sauteur. Elle montre ensuite que la sélection sur performances semble devoir être préférée à une sélection indirecte sur le modèle. Dans certains cas toutefois, l'information apportée par les mensurations corporelles peut constituer un complément d'appréciation non négligeable qui doit être préféré à l'œil de l'expert souvent trompé par l'état de l'animal ou l'art du présentateur.

Rę̧u pour publication en décembre 1978 .

\section{Summary}

\section{Analysis of relationships between morphology and gallop, trot and jumping abilities in horses}

In order to easily obtain a large number of measures per horse we tested an improved photo metric method. The estimations of the repeatability of about thirty measures made on 60 horses show that two or three photographies are sufficient to obtain relatively precise estimations. On the other hand, component analysis show four main factors: the height (4o p. roo of the total variance), compactness (I 2 p. roo), directions of croup and hindlegs (7 p. Ioo), direction of shoulders and forelegs $(6 \mathrm{p}$. IOO).

Study of the differences between French Trotters and Thoroughbreds was made with this method on $4^{\circ}$ and 42 animals, respectively. Analysis between theses two populations led to an accurate determination of the amount of divergencies induced in these two breeds by selection on two different types of races. Trotters are characterized by larger ilium, femur and scapulum angles relative to the horizontal line. The radius is shorter, canon bone markedly thicker. In addition to this, French Trotters have the same wither height as Thoroughbreds, but they are broader and therefore more compact.

The relationship between conformation and jumping ability in show horses was studied on two independent samples:

The first one was represented by 94 stallions of national studs, progeny tested in jumping shows with a determination coefficient of $R^{2} \geqslant 0.40$.

The second one was represented by 103 horses divided into two groups, "Good " and "Bad jumpers ", chosen among 26 riding establishments.

Explanation of the selection index by polynomial regression is rather disappointing. At the $5 \mathrm{p}$. Ioo level, the wither height is the only variable having a significant contribution in the whole sample (94 stallions) as well as in the French Saddle Breed sample (45 stallions). In Angloarabians (28 stallions) a small humerus angle to the horizontal line seems to be preferable. In Thorouggbreds (2I stallions) a large ilium angle to the horizontal line is the most suitable with jumping ability.

In the second sample, a two-way analysis of variance (sex and jumping ability) was applied. Nine measures were significantly affected. "Good jumpers " are characterized by a larger width at the shoulders, hips and trochanters as well as a larger chest girth. For the hind legs, the ilium is larger and the femur angle to the horizontal line smaller.

The discriminant analysis permits to easily distinguish a trotter from a thoroughbred ( $5 \mathrm{p}$. Ioo of missclassed), but it underlines the difficulty of distinguishing a "good jumper " from a "bad one" (27 p. Ioo of missclassed).

However, trends can be deduced from the overall calculations. The jumper is a rather tall horse, very compact (broad and near the ground), with a long and broad croup, strongly inclined and with rather low scapulum, humerus and femur angles to the horizontal line. 
In order to check the efficiency of " type and gait" trials in selecting for sport, performance test indexes in jumping shows of 645 horses were compared to ranking of the same horses in the regional trial of Saint-Lô from 1971 to 1975 . Correlation coefficients showed that " type and gait " trials only explain 4 p. I 00 of the breeding value for jumping ability while the individual performances based on earnings explain about $20 \mathrm{p}$. Ioo and progeny testing of stallions $60 \mathrm{p}$. 100, on an average.

It may be concluded that for genetic improvement of jumping ability the performance test is much more efficient than the indirect selection on body type and gaits.

\section{Références bibliographiques}

BENARD D., I968. Étude analytique de la conformation du cheval de Selle Français à partir d'un échantillon de sa population. Mémoire de fin d'études de l'E.S.I.T.P.A. polycopié C.N.R.Z.I.N.R.A. Jouy-en-Josas.

BURGER H. J., 1971. Structure généalogique de l'effectif des chevaux d'élevage et des chevaux de course dans les Haras Nationaux de Lindenhof et de Prieros, compte tenu en particulier de l'extérieur et des performances de course et d'élevage. Mémoire de fin d'études, Université F. Schiller, Iena (Ger).

Dusek J., Ehrlein H. J., Engelhardt (Von) W. H., Hornicke H., 1970. Corrélation entre la longueur du pas et la vitesse chez les chevaux. Z. Tierzucht Zücht. Biol., 87, I77-I88.

Fabiani M., I972, 1973 et 1974. Première évaluation de l'aptitude à l'obstacle des chevaux I. Chevaux des établissements d'entraînement pour l'exportation. Instytut Genetyki, Hodowli Zwierzat Polskief Academii Nauk Biulutyn 1972, 27, 35-44.

II. Chevaux des établissements d'entraînement de Kwidzyu et de Bialy Bor. Prace i materialy zootechniczne, 1973, 4, 39-54.

III. Chevaux des établissements de course appartenant à l'état de Varsovie. Prace $i$ materialy zootechniczne, 1974, 5, 41-55.

Fabiani M., I975. Étude de quelques caractères des chevaux gagnants entre r950 et r97I et résultats pour une approximation de l'évaluation de l'aptitude au saut d'obstacles. Prace $i$ materialy zootechniczne, 9, 107-121.

FroIdevaux J., 1975. Aspects méthodologiques de l'étude des mensurations corporelles chez le Cheval. Mémoire de fin d'études de 1'E.S.I.T.P.A. Polycopié C.N.R.Z.-I.N.R.A. Jouy-en-Josas.

Gaste (de) M., I908. "Le modèle et les allures ". Librairie Milon, J. B. Robert, successeur, Saumur.

Goubaux A., Barrier G., I 884. De l'extérieur du Cheval. Asselin et Cie Éditeur, Paris, I o67 p.

LAMARCHE L., 1977. Étude de la liaison entre la morphologie et l'aptitude au saut d'obstacles chez le cheval de sport. Mémoire de fin d'études de l'E.S.I.T.P.A., Polycopié C.N.R.Z.-I.N.R.A. Jouy-en-Josas.

I.ANGLOIS B., r977. L,e classement des étalons sur leur descendance. Le classement des juments sur leurs performances. L'éperon, (125), 44-46.

L.EGault P., 1977. Contribution à l'étude de la morphologie comparée des chevaux de Pur Sang Anglais et Trotteurs Français. Thèse E.N.V. Alfort. Université de Paris.

LEMOIGNE A., I877. Recueil de médecine vétérinaire, I877, 81-208.

LuSH J. I., I931. J. Agric. Res. 853. Cité d'après Snedecor et Cochran, r97 I, I86-I 88.

NEISSER E., I976. Corrélation entre phénotype et performances chez le cheval de sport. Archiv. für Tierzucht, 19, (I), 5I-6o.

Pearson E. S., Hartiey H. O., r972. Biometrika Tables for Statisticians. Cambridge University Press Published for the Biometrika Trustees. Vol. 2 p. 27-31 et Table 9 p. 205-210.

SCHEIDE J., 1968. Structure généalogique des troupeaux de juments dans les haras d'état de Hoppegarten et de Neustadt, compte tenu en particulier des relations entre l'extérieur et les performances. Mémoire de fin d'études, Université F. Schiller, Iena (Ger).

Schwark H. J., Scherber K., SASSE L., 1977. Corrélations entre caractéristiques chez le cheval de sport. Fédération Européenne de Zootechnie, Bruxelles 22-25 août 1977. (Commission chevaline).

Snedecor G. W., Cochran W. G., 1971. Statistical Methods. Sixième édition - Iowa State University Press. Ames Iowa, U.S.A.

Spearman C., r904. Amer. J. Psych. I5-88 cité d'après Snedecor et Cochran, I971, r93-r95. 